\title{
Apolipoprotein E $\varepsilon 4$ Polymorphism as a Risk Factor for Ischemic Stroke: A Systematic Review and Meta-Analysis
}

\author{
Su-Ya Qiao, Ke Shang, Yun-Hui Chu, Hai-Han Yu, Xin Chen, Chuan Qin (D), Deng-Ji Pan (D), \\ and Dai-Shi Tian
}

Department of Neurology, Tongji Hospital, Tongji Medical College, Huazhong University of Science and Technology, Wuhan 430030, China

Correspondence should be addressed to Chuan Qin; qinchuan712@126.com, Deng-Ji Pan; djpan@tjh.tjmu.edu.cn, and Dai-Shi Tian; tiands@tjh.tjmu.edu.cn

Received 25 October 2021; Revised 21 December 2021; Accepted 5 January 2022; Published 3 February 2022

Academic Editor: XIANWEI ZENG

Copyright (c) 2022 Su-Ya Qiao et al. This is an open access article distributed under the Creative Commons Attribution License, which permits unrestricted use, distribution, and reproduction in any medium, provided the original work is properly cited.

Introduction. Rising studies indicate that the apolipoprotein E (APOE) gene is related to the susceptibility of ischemic stroke (IS). However, certain consensus is limited by the lack of a large sample size of researches. This meta-analysis was performed to explore the potential association between the APOE gene and IS. Methods. To identify relevant case control studies in English publications by October 2020, we searched PubMed, Embase, Web of Science, and the Cochrane Library. Pooled odds ratios (ORs) with fixedor random-effect models and corresponding 95\% confidence intervals (CIs) were calculated to analyze potential associations. Results. A total of 55 researches from 32 countries containing 12207 IS cases and 27742 controls were included. The association between APOE gene $\varepsilon 4$ mutation and IS was confirmed ( $\varepsilon 4$ vs. $\varepsilon 3$ allele: pooled OR $=1.374,95 \%$ CI, 1.214-1.556; $\varepsilon 2 / \varepsilon 4$ vs. $\varepsilon 3 / \varepsilon 3$ : pooled $\mathrm{OR}=1.233,95 \% \mathrm{CI}, 1.056-1.440 ; \varepsilon 3 / \varepsilon 4$ vs. $\varepsilon 3 / \varepsilon 3$ : pooled $\mathrm{OR}=1.340,95 \% \mathrm{CI}, 1.165-1.542 ; \varepsilon 4 / \varepsilon 4$ vs. $\varepsilon 3 /$ $\varepsilon 3$ : pooled $\mathrm{OR}=1.833,95 \% \mathrm{CI}, 1.542-2.179$; and $\mathrm{APOE} \varepsilon 4$ carriers vs. non- $\varepsilon 4$ carriers: pooled $\mathrm{OR}=1.377 ; 95 \% \mathrm{CI}, 1.203-$ $1.576)$. Interestingly, APOE $\varepsilon 4$ mutation showed a dose-response correlation with IS risk $(\varepsilon 4 / \varepsilon 4$ vs. $\varepsilon 2 / \varepsilon 4:$ pooled OR $=1.625$; $95 \% \mathrm{CI}, 1.281-2.060 ; \varepsilon 4 / \varepsilon 4$ vs. $\varepsilon 3 / \varepsilon 4$ : pooled $\mathrm{OR}=1.301 ; 95 \% \mathrm{CI}, 1.077-1.571)$. Similar conclusions were drawn in the small artery disease (SAD) subtype, but not in large artery atherosclerosis (LAA) or in cardioaortic embolism (CE), by subgroup analysis. Conclusions. These observations reveal that specific APOE $\varepsilon 4$ mutation was significantly associated with the risk of IS in a dose-dependent manner, while APOE $\varepsilon 4$ mutation was related to SAD subtype onset without a cumulative effect.

\section{Introduction}

Ischemic stroke (IS) is a disturbing problem worldwide, which is attributable to its leading role in disability and mortality worldwide, regardless of age, ethnicity, or gender [1]. Uncovering the etiology of IS is crucial for recognition and prevention of this disorder. Genetic elements and environmental components positively contribute to this multifactorial disease $[2,3]$. Genetic inheritance provides a guide to the identification of high-risk individual. It deserves to investigate candidate gene polymorphisms in IS pathophysiological pathways. The apolipoprotein E (APOE) gene locates on chromosome 19q13.2. Two single polymorphisms (rs7412 and rs729358), three common alleles $(\varepsilon 2, \varepsilon 3$, and $\varepsilon 4)$, and six genotypes $(\varepsilon 2 / \varepsilon 2, \varepsilon 2 / \varepsilon 3, \varepsilon 2 / \varepsilon 4, \varepsilon 3 / \varepsilon 3, \varepsilon 3 / \varepsilon 4$, and $\varepsilon 4 / \varepsilon 4)$ generate in populations [4]. The product of the APOE gene is a polymorphic protein named apolipoprotein $\mathrm{E}$, which modulates the translocation of the cholesterol and other lipids among highly diverse cells [5], involved with neuroinflammation [6] and myelin integrity maintenance [7]. A study indicated that the activated CypA-MMP9 pathway in APOE4 carriers facilitated pericyte injury, which caused blood vessel dysfunction [8]. APOE polymorphisms and its risk associations with coronary artery disease [9], hypertension [10], diabetes [11], and carotid arterial atherosclerosis [12] are widely debated. The abovementioned diseases place individuals at a potential serious risk of IS. Individual studies of the association between IS and APOE polymorphisms have been explored extensively. Clinical differences, ethnic diversities, and small sample sizes restricted the present 
finding to an inconsistent and controversial one. Previous meta-analyses concerning to this issue have been published several years ago [13] or limited to specific ethnicity [14, 15]. Accordingly, researches from 32 countries are qualified to form our meta-analysis to clarify how APOE genotypes are associated with IS. Moreover, we firstly revealed the correlation of the APOE gene and three IS subtypes (large artery atherosclerosis (LAA), small artery disease (SAD), and cardioaortic embolism (CE)).

\section{Materials and Methods}

We followed the rules of the preferred reporting items for systematic reviews and meta-analyses (PRISMA) statement to make this meta-analysis [16].

2.1. Data Availability. The data that contribute to the findings in our study are available and the corresponding authors can be contacted for data access.

2.2. Literature Search. Online databases (PubMed, Embase, Web of Science, and the Cochrane Library) were comprehensively searched for studies potentially involved and published in English publications and prior to October 30, 2020. We used a combination of some search terms relevant for IS (stroke, cerebral infarct, brain infarct, ischemic stroke, cerebral ischemia, transient ischemic attack, and cerebrovascular accident) and for the APOE gene (apolipoprotein E, APOE polymorphisms, apolipoprotein E polymorphisms, apolipoprotein E gene, rs429358, rs7412, apolipoprotein E epsilon 4, APOE e4, apolipoprotein E epsilon 2, and APOE e2). The detailed search strategies were showed next.

2.3. Selection Criteria. The selection of the studies was independently completed by two investigators, and any difference was resolved by discussion until an agreement was reached. We carefully selected case control studies that evaluated the relationship of the APOE gene and IS with definite IS diagnoses (using computed tomography, magnetic resonance, or autopsy) regardless of the ethnic background. The detailed inclusion criteria were (1) high-quality studies which explore the relationship between the APOE gene and IS, (2) explicit IS diagnostic criteria, (3) nonstroke individuals as the control group, and (4) original data including independent and sufficient APOE genotype data, to compute ORs and 95\% CIs. The newest and largest studies were chosen to avoid duplicate or overlapped data information.

2.4. Data Extraction. Two investigators separately finished full-text reading to extract the needed information from each selected study and resolved the controversial items through serious discussion. The extracted information was (1) research characteristics, including the first author's name, year of publication, and geographical location of the study; (2) participant details, such as the sex ratio, mean age, and the sample size of case and control groups; (3) diagnostic criteria for IS; (4) determination methods of the APOE gene; (5) each genotype frequency; (6) the sample sizes of IS subtypes according to TOAST norms and respective genotype frequency; and (7) HWE in controls.
2.5. Quality Assessment. We performed the quality assessment through the Newcastle-Ottawa Scale (NOS) score considering selection, comparability, and exposure. It ranged from 0 (worst) to 9 (best) and high-quality studies were known as with a NOS score $\geq 7$.

2.6. Statistical Analysis. We performed Stata 14.0 to complete all data analyses. The chi-square test was used to examine the Hardy-Weinberg equilibrium (HWE) in control groups. An overt deviation from $\mathrm{HWE}$ was regarded as $P$ $<0.05$. The compositive ORs and 95\% CI were calculated. We explored five genetic models to generate the respective pooled ORs: (1) allele comparisons ( $\varepsilon 2$ allele vs. $\varepsilon 3$ allele; $\varepsilon 4$ allele vs. $\varepsilon 3$ allele); (2) genotype comparisons ( $\varepsilon 2 / \varepsilon 2$ vs. $\varepsilon 3 / \varepsilon 3 ; \varepsilon 2 / \varepsilon 3$ vs. $\varepsilon 3 / \varepsilon 3 ; \varepsilon 2 / \varepsilon 4$ vs. $\varepsilon 3 / \varepsilon 3 ; \varepsilon 3 / \varepsilon 4$ vs. $\varepsilon 3 / \varepsilon 3 ; \varepsilon 4 / \varepsilon 4$ vs. $\varepsilon 3 / \varepsilon 3$ ); (3) APOE $\varepsilon 4$ carrier comparisons: we defined three $\varepsilon 4$-containing genotypes $(\varepsilon 2 / \varepsilon 4+\varepsilon 3 / \varepsilon 4+\varepsilon 4 / \varepsilon 4)$ as APOE $\varepsilon 4$ carriers and the other genotypes $(\varepsilon 2 / \varepsilon 2+\varepsilon 2 / \varepsilon 3+\varepsilon 3 / \varepsilon 3)$ as non-APOE $\varepsilon 4$ carriers; (4) APOE $\varepsilon 2$ carrier comparisons: similar comparisons of $\varepsilon 2$-containing genotypes $(\varepsilon 2 / \varepsilon 2+\varepsilon 2 / \varepsilon 3+\varepsilon 2 / \varepsilon 4)$ vs. non- $\varepsilon 2$-containing genotypes $(\varepsilon 3 / \varepsilon 3+\varepsilon 3 / \varepsilon 4+\varepsilon 4 / \varepsilon 4)$; and (5) comparisons between APOE $\varepsilon 4$ homozygosis and $\varepsilon 4$ heterozygote $(\varepsilon 4 / \varepsilon 4$ vs. $\varepsilon 2 / \varepsilon 4 ; \varepsilon 4 / \varepsilon 4$ vs. $\varepsilon 3 / \varepsilon 4)$. The $I^{2}$ statistic and Cochran's $Q$ test were applied to measure the heterogeneity between studies [17]. We selected the random effect model (DerSimonian-Laird method) when heterogeneity was found between studies $\left(I^{2}>50.0 \%\right)$ and fixed-effect model (Mantel-Haenszel method) when no heterogeneity existed $\left(I^{2}<50.0 \%\right)$. Subgroup analysis was conducted to confirm the relationship between the APOE polymorphisms and the risk of different IS subgroups. Sensitivity analysis was performed by successively removing a single study one by one to verify the stability and reliability of our conclusions. Meta-regression analysis was operated to recognize sources of heterogeneity. Funnel plots and quantified Egger's tests were accomplished to test publication bias. Significant publication bias was considered as the $P$ value of Egger's test less than 0.10 or obvious asymmetric funnel plot.

2.7. The Result of Trial Sequential Analysis (TSA). Insufficient sample size, continuous updating, and repeating "significance testing" could increase the risk of type I errors. Therefore, traditional meta-analysis that focuses on the specific topic may suffer an increased risk of random error. Trial sequential analysis (TSA) was used to reduce the risk of type I error and obtain important information regarding the required sample size for such trials. Set the time sequence of a single study as the research node, and then, perform an interim analysis between the new study that will be included in meta-analysis and existing data accumulation. The required information size (RIS), trial sequential monitoring boundary, and futility boundary are estimated using the TSA. As the sample size of meta-analysis reaching the RIS or the $z$-curve crossing the trial sequential monitoring boundary, we can conclude that the results of metaanalysis are quite stable and further studies were not needed. We accomplished TSA following the guidelines of the user manual and previous article [18] by setting a 




Figure 1: A flow diagram of identification and selection process of the included literatures in this meta-analysis.

significance of $5 \%$ for type I error, a relative risk reduction of $20 \%$, and a statistical test power of $80 \%$ with TSA software (TSA, version 0.9 beta; Copenhagen Trial Unit, Copenhagen, Denmark).

\section{Results}

3.1. Characteristics of Eligible Studies. We collect a total of 55 studies from 32 countries containing 12207 IS cases and 27742 controls to make the meta-analysis [19-73]. Figure 1 showed the detailed selection process. The selected studies and their main characteristics were exhibited in Table 1. Fifteen of the studies provided data about different subtypes (grouped by classification of cerebrovascular diseases III or TOAST classification) of IS: large artery atherosclerosis (LAA), small artery disease (SAD), and cardioaortic embolism (CE). We extracted them independently and specific information was showed in supplementary material table 1. There were seven studies (Koopal et al. 2016, Lai et al. 2007, Chowdhury et al. 2001, Kokubo et al. 2000, Ji et al. 1998, Couderc et al. 1993, Saidi et al. 2009) which deviated HWE obviously, and one study (Schneider et al. 2005) did not contain enough data to obtain HWE. Forty-eight studies used PCR-based method and seven researches (Slowik et al. 2003, Karttunen et al. 2002, Hachinski et al. 1996, Couderc et al. 1993, Brewin et al. 2020, Aalto-Setala et al. 1998, Schneider et al. 2005) used other methods to identify APOE genotypes. These studies used computed tomography or magnetic resonance to diagnose IS except that one research which used autopsy (Schneider et al. 2005). The NOS score mean value was 7.509 , which suggested that the quality of included studies was reliable (supplementary material Table 2). PRISMA2020 checklist was provided to present our meta-analysis items (supplementary material Table 3).

\subsection{Main Results of the Comparisons in the Abovementioned Five Genetic Models}

3.2.1. Allele Comparisons. In comparison with the $\varepsilon 3$ allele, the $\varepsilon 2$ allele did not show association of the risk of IS (pooled $\mathrm{OR}=0.983,95 \% \mathrm{CI}, 0.867-1.115, P=0.79$ ) (as showed in Table 2), while the $\varepsilon 4$ allele contributed to an obviously increased risk of IS with the pooled OR $=1.374$ (95\% CI, 1.214-1.556, $P<0.0001$ ) (Figure 2(d)).

3.2.2. Genotype Comparisons. When compared with the $\varepsilon 3 /$ $\varepsilon 3$ genotype, the pooled effects of the APOE genotype in the meta-analysis were as follows: for the $\varepsilon 2 / \varepsilon 2$ genotype, pooled $\mathrm{OR}=0.985,95 \% \mathrm{CI}, 0.653-1.486, P=0.94$, and for the $\varepsilon 2 / \varepsilon 3$ genotype, pooled $\mathrm{OR}=0.980,95 \% \mathrm{CI}, 0.900$ $1.066, P=0.63$; those two genotypes presented no association with the risk of IS (as showed in Table 2). Genotypes $\varepsilon 2 / \varepsilon 4, \varepsilon 3 / \varepsilon 4$, and $\varepsilon 4 / \varepsilon 4$ were related to a higher risk of IS than $\varepsilon 3 / \varepsilon 3$. The respective IS risk ORs were 1.233 (95\% CI, 1.056-1.440, $P=0.01$ ) (Figure 2(a)), 1.340 (95\% CI, 1.1651.542, $P<0.0001$ ) (Figure 2(b)), and 1.833 (95\% CI, 1.5422.179, $P<0.0001$ ) (Figure $2(\mathrm{c})$ ). The above results could be found in Table 2. A conclusion was drawn: every genotype which contained APOE $\varepsilon 4$ mutation increased the risk of IS.

3.2.3. APOE \&4 Carrier Comparisons. Compared with the non- $\varepsilon 4$ carriers, we confirmed that the $\varepsilon 4$ carriers were associated with the increased risk of IS; the pooled outcome was pooled OR $=1.377$ (95\% CI, 1.203-1.576, $P$ $<0.0001$ ) (Figure 2(e)). 


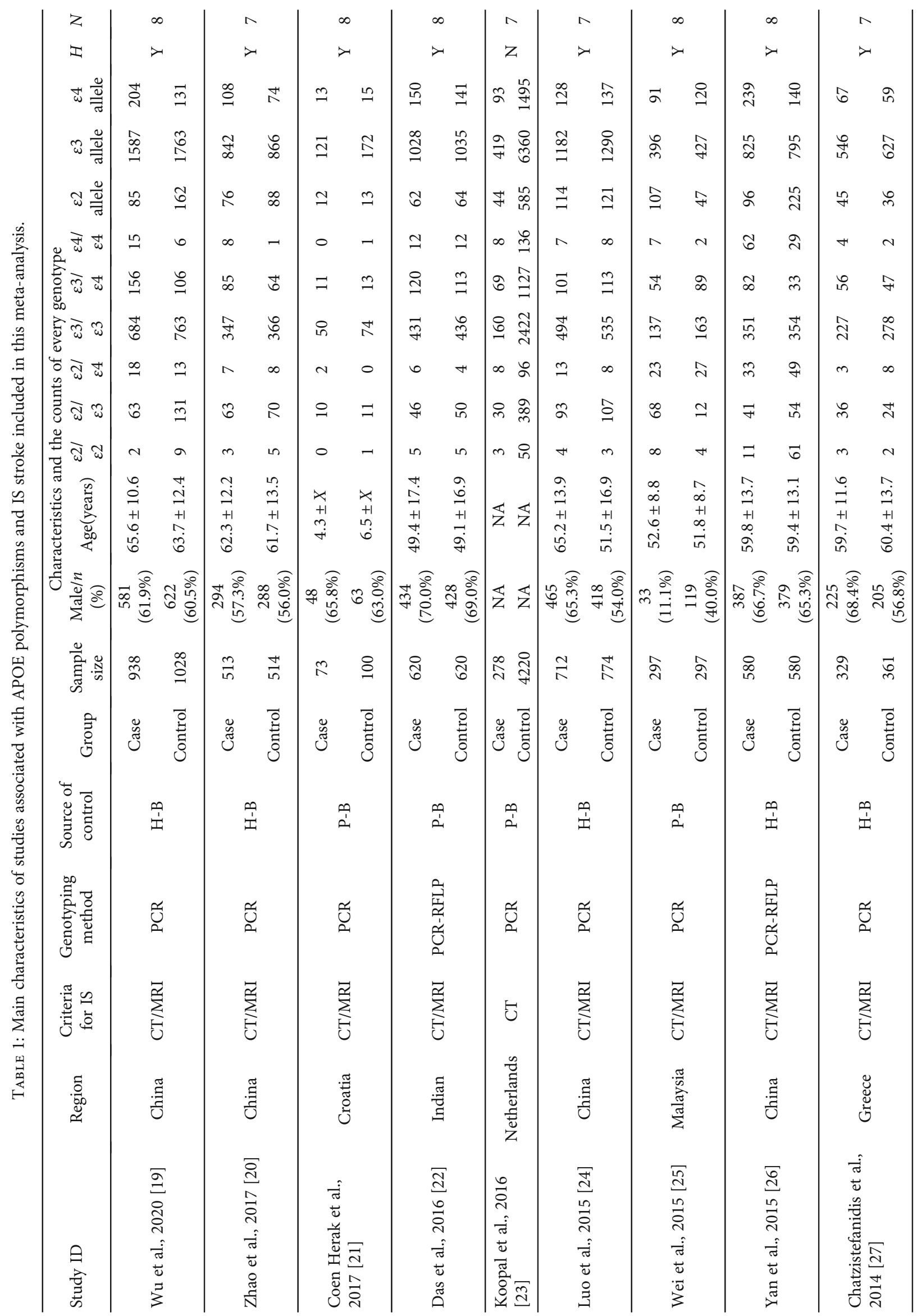




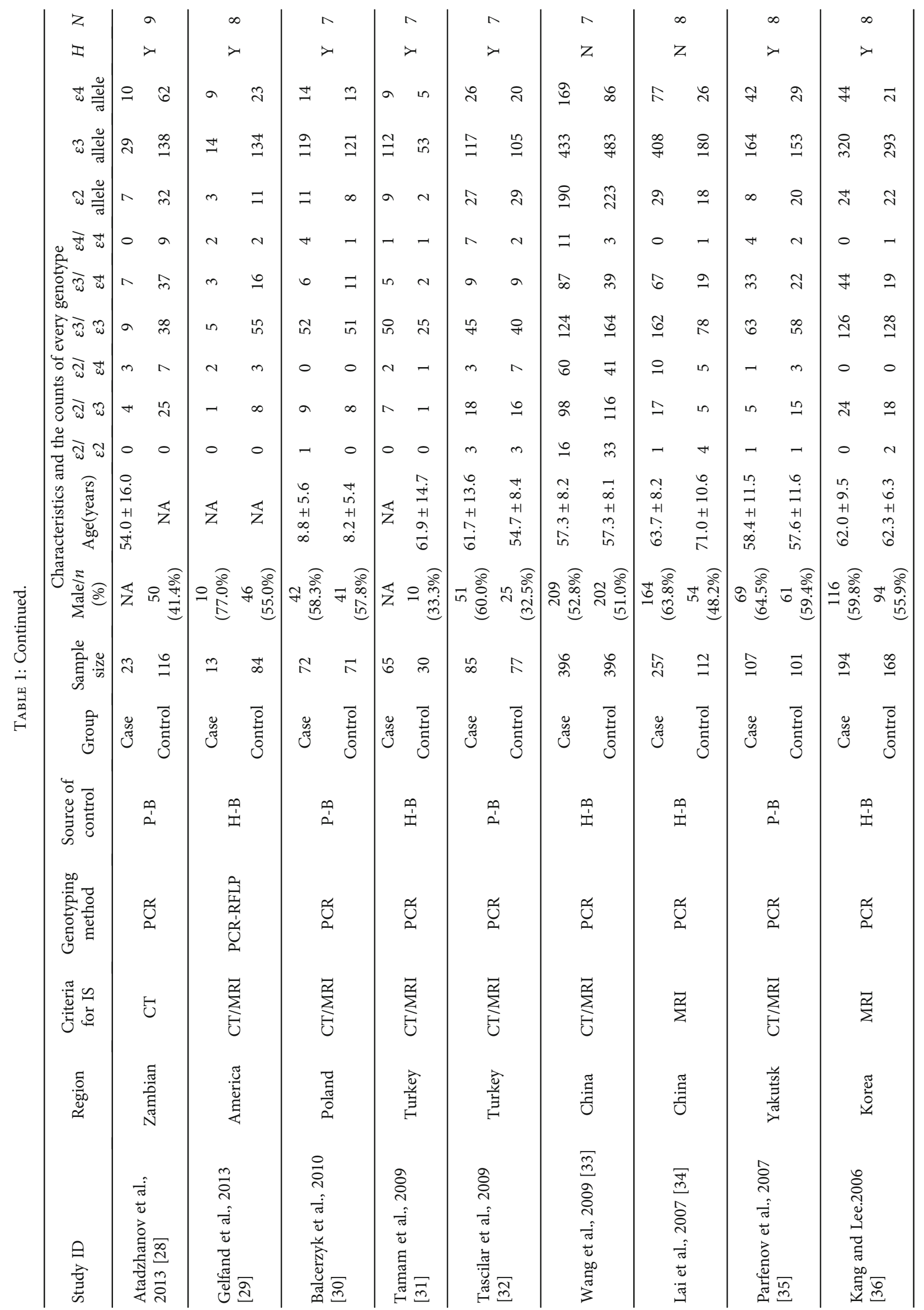




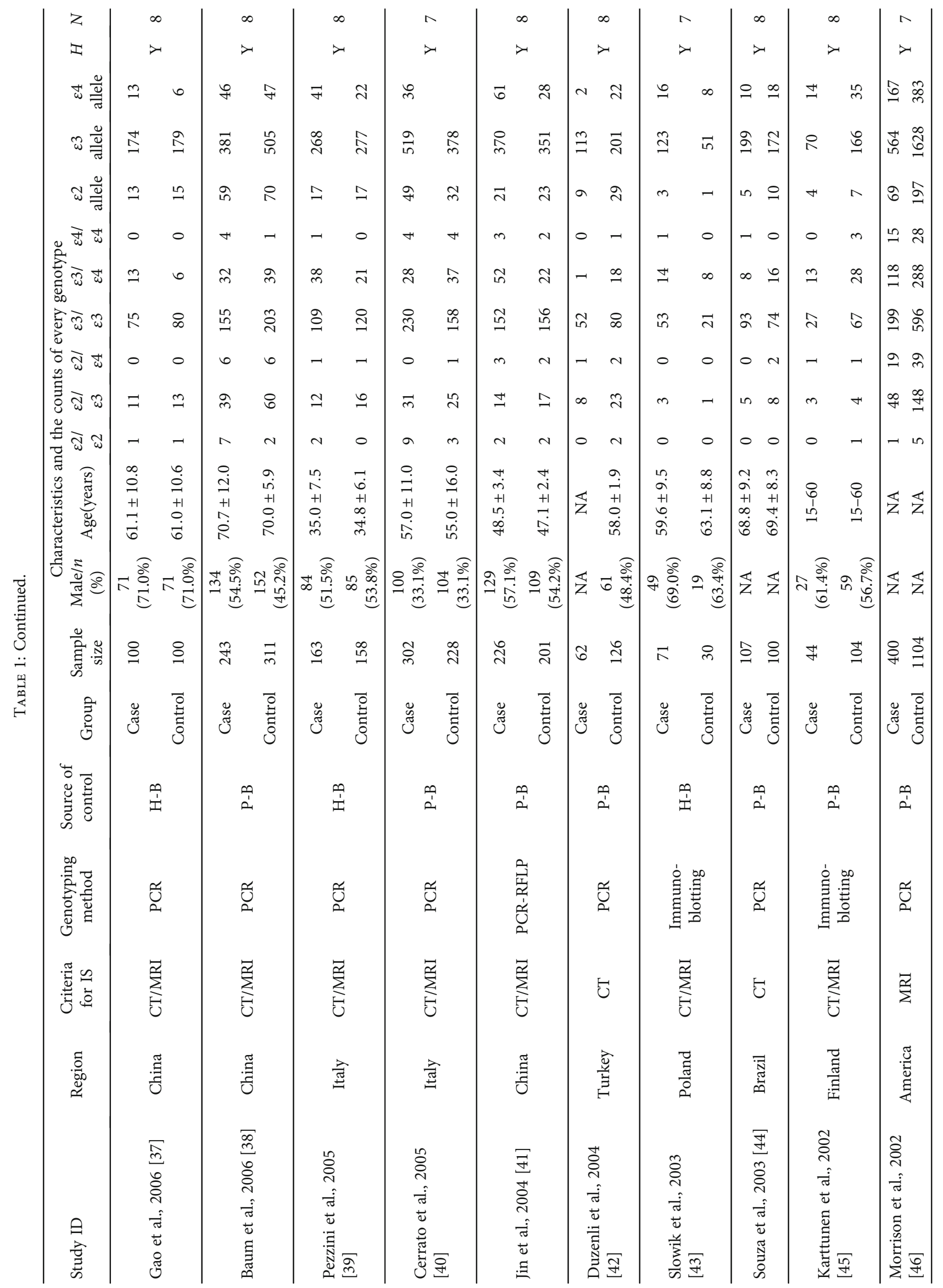




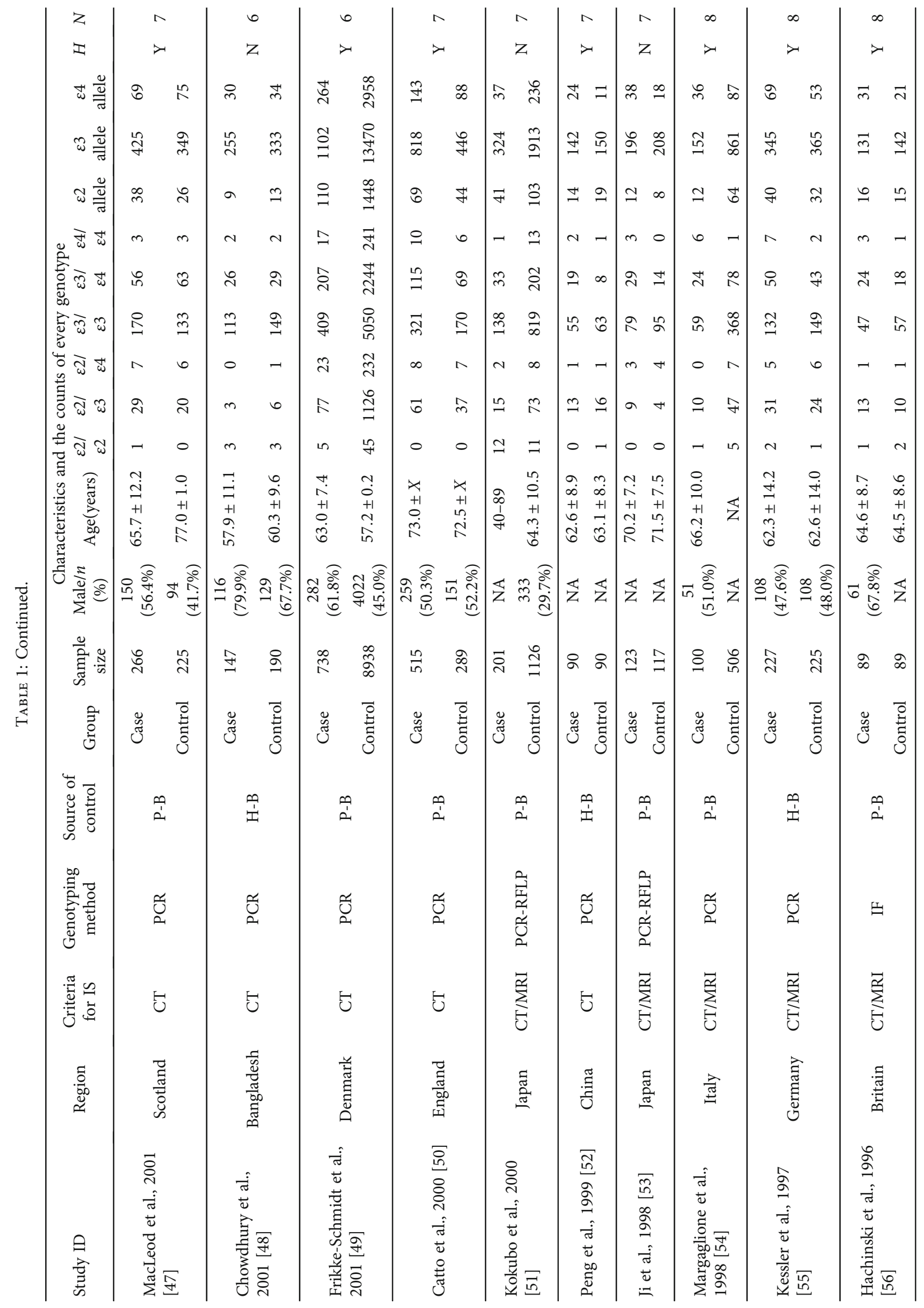




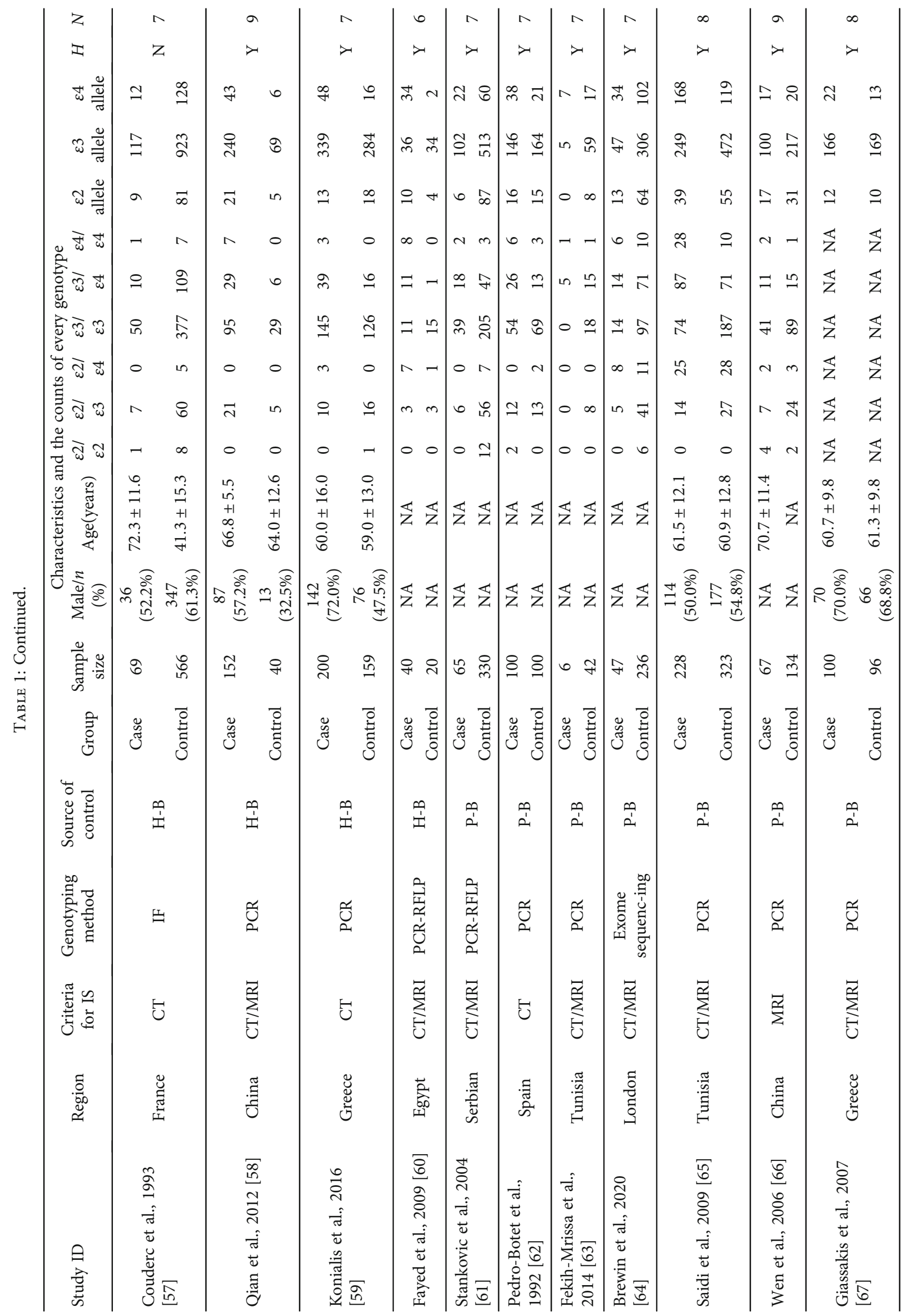




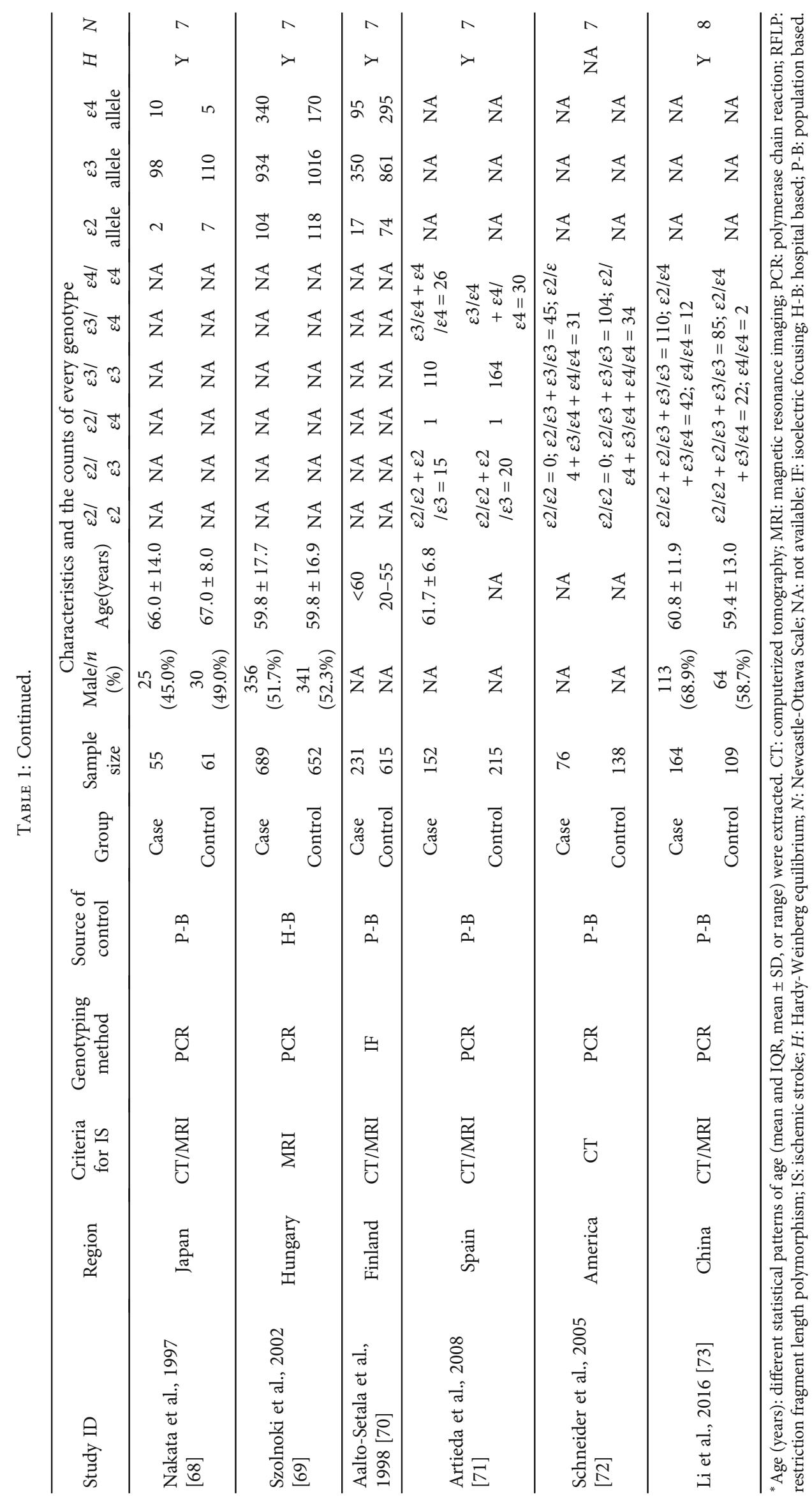


TABLE 2: The main results of the APOE gene associated with IS included in the meta-analysis.

\begin{tabular}{|c|c|c|c|c|c|}
\hline \multirow{2}{*}{ Genetic model of APOE gene polymorphisms } & \multirow{2}{*}{ Group } & \multirow{2}{*}{ No. of included studies } & \multicolumn{3}{|c|}{ Results of association with IS } \\
\hline & & & OR & $95 \% \mathrm{CI}$ & $P$ value of ORs \\
\hline \multirow{4}{*}{$\varepsilon 2$ allele vs. $\varepsilon 3$ allele } & All & 51 & 0.983 & $(0.867,1.115)$ & 0.79 \\
\hline & LAA & 13 & 0.962 & $(0.712,1.299)$ & 0.80 \\
\hline & $\mathrm{CE}$ & 10 & 1.517 & $(0.861,2.674)$ & 0.15 \\
\hline & SAD & 12 & 1.190 & $(0.997,1.421)$ & 0.05 \\
\hline \multirow{4}{*}{$\varepsilon 4$ allele vs. $\varepsilon 3$ allele } & All & 51 & 1.374 & $(1.214,1.556)$ & $<0.0001$ \\
\hline & LAA & 13 & 1.149 & $(0.898,1.469)$ & 0.27 \\
\hline & $\mathrm{CE}$ & 10 & 1.092 & $(0.662,1.801)$ & 0.73 \\
\hline & SAD & 12 & 1.318 & $(1.073,1.618)$ & 0.01 \\
\hline \multirow{4}{*}{$\varepsilon 2 / \varepsilon 2$ vs. $\varepsilon 3 / 3$} & All & 36 & 0.985 & $(0.653,1.486)$ & 0.94 \\
\hline & LAA & 11 & 1.307 & $(0.750,2.278)$ & 0.35 \\
\hline & $\mathrm{CE}$ & 10 & 4.290 & $(1.917,9.600)$ & $<0.0001$ \\
\hline & SAD & 11 & 1.803 & $(1.037,3.134)$ & 0.04 \\
\hline \multirow{4}{*}{$\varepsilon 2 / \varepsilon 3$ vs. $\varepsilon 3 / 3$} & All & 46 & 0.980 & $(0.900,1.066)$ & 0.63 \\
\hline & LAA & 13 & 0.869 & $(0.705,1.071)$ & 0.19 \\
\hline & $\mathrm{CE}$ & 10 & 1.255 & $(0.849,1.856)$ & 0.26 \\
\hline & SAD & 12 & 1.178 & $(0.952,1.457)$ & 0.13 \\
\hline \multirow{4}{*}{$\varepsilon 2 / \varepsilon 4$ vs. $\varepsilon 3 / 3$} & All & 42 & 1.233 & $(1.056,1.440)$ & 0.01 \\
\hline & LAA & 11 & 0.978 & $(0.607,1.576)$ & 0.93 \\
\hline & $\mathrm{CE}$ & 10 & 1.458 & $(0.534,3.980)$ & 0.46 \\
\hline & SAD & 10 & 0.932 & $(0.526,1.652)$ & 0.81 \\
\hline \multirow{4}{*}{$\varepsilon 3 / \varepsilon 4$ vs. $\varepsilon 3 / 3$} & All & 47 & 1.340 & $(1.165,1.542)$ & $<0.0001$ \\
\hline & LAA & 14 & 1.154 & $(0.841,1.584)$ & 0.38 \\
\hline & $\mathrm{CE}$ & 10 & 1.175 & $(0.627,2.203)$ & 0.62 \\
\hline & SAD & 13 & 1.392 & $(1.097,1.767)$ & 0.01 \\
\hline \multirow{4}{*}{$\varepsilon 4 / \varepsilon 4$ vs. $\varepsilon 3 / 3$} & All & 46 & 1.833 & $(1.542,2.179)$ & $<0.0001$ \\
\hline & LAA & 13 & 1.367 & $(0.836,2.236)$ & 0.21 \\
\hline & $\mathrm{CE}$ & 10 & 1.543 & $(0.591,4.029)$ & 0.38 \\
\hline & SAD & 11 & 1.809 & $(1.030,3.175)$ & 0.04 \\
\hline \multirow{4}{*}{$\varepsilon 4$ vs. non- $\varepsilon 4$} & All & 50 & 1.377 & $(1.203,1.576)$ & $<0.0001$ \\
\hline & LAA & 14 & 1.149 & $(0.876,1.506)$ & 0.32 \\
\hline & $\mathrm{CE}$ & 10 & 1.091 & $(0.645,1.845)$ & 0.74 \\
\hline & SAD & 13 & 1.329 & $(1.064,1.661)$ & 0.01 \\
\hline \multirow{4}{*}{$\varepsilon 2$ vs. non- $\varepsilon 2$} & All & 48 & 0.956 & $(0.841,1.086)$ & 0.49 \\
\hline & LAA & 14 & 0.861 & $(0.717,1.035)$ & 0.11 \\
\hline & $\mathrm{CE}$ & 10 & 1.358 & $(0.966,1.910)$ & 0.08 \\
\hline & SAD & 13 & 1.117 & $(0.926,1.347)$ & 0.25 \\
\hline \multirow{4}{*}{$\varepsilon 4 / \varepsilon 4$ vs. $\varepsilon 2 / 4$} & All & 40 & 1.625 & $(1.281,2.060)$ & $<0.0001$ \\
\hline & LAA & 11 & 1.551 & $(0.791,3.043)$ & 0.20 \\
\hline & $\mathrm{CE}$ & 9 & 0.771 & $(0.177,3.352)$ & 0.73 \\
\hline & SAD & 4 & 2.115 & $(0.919,4.867)$ & 0.08 \\
\hline \multirow{4}{*}{$\varepsilon 4 / \varepsilon 4$ vs. $\varepsilon 3 / 4$} & All & 46 & 1.301 & $(1.077,1.571)$ & 0.01 \\
\hline & LAA & 13 & 1.353 & $(0.811,2.258)$ & 0.25 \\
\hline & $\mathrm{CE}$ & 6 & 1.077 & $(0.402,2.887)$ & 0.88 \\
\hline & SAD & 11 & 1.332 & $(0.739,2.400)$ & 0.34 \\
\hline
\end{tabular}






(a)

FIgUre 2: Continued. 


\begin{tabular}{|c|c|c|}
\hline $\begin{array}{l}\text { Study } \\
\text { ID }\end{array}$ & OR $(95 \% \mathrm{CI})$ & $\begin{array}{l}\% \\
\text { Weight }\end{array}$ \\
\hline Wu et al. 2020 & $1.642(1.256,2.145)$ & 3.37 \\
\hline Gelfand et al. 2013 & $2.063(0.444,9.580)$ & 0.69 \\
\hline Coen Herak et al. 2017 & $1.252(0.520,3.018)$ & 1.53 \\
\hline Jin et al. 2004 & $2.426(1.405,4.189)$ & 2.43 \\
\hline Luo et al. 2015 & $0.968(0.721,1.300)$ & 3.29 \\
\hline Pedro-Botet et al. 1992 & $2.556(1.201,5.437)$ & 1.82 \\
\hline Baum et al. 2006 & $1.075(0.644,1.793)$ & 2.54 \\
\hline Yan et al. 2015 & $2.506(1.630,3.853)$ & 2.82 \\
\hline Chatzistefanidis et al. 2014 & $1.459(0.953,2.233)$ & 2.84 \\
\hline Atadzhanov et al. 2013 & $0.799(0.270,2.368)$ & 1.16 \\
\hline Zhao et al. 2017 & $1.401(0.981,2.000)$ & 3.08 \\
\hline Balcerzyk et al. 2010 & $0.535(0.184,1.555)$ & 1.19 \\
\hline Tamam et al. 2009 & $1.250(0.226,6.902)$ & 0.57 \\
\hline Tascilar et al. 2009 & $0.889(0.321,2.459)$ & 1.27 \\
\hline Wang et al. 2009 & $2.950(1.893,4.599)$ & 2.78 \\
\hline Lai et al. 2007 & $1.698(0.954,3.022)$ & 2.33 \\
\hline Peng et al. 1999 & $2.720(1.104,6.703)$ & 1.49 \\
\hline Karttunen et al. 2002 & $1.152(0.520,2.552)$ & 1.72 \\
\hline Duzenli et al. 2004 & $0.085(0.011,0.660)$ & 0.42 \\
\hline Frikke-Schmidt et al. 2001 & $1.139(0.957,1.356)$ & 3.63 \\
\hline Pezzini et al. 2005 & $1.992(1.101,3.603)$ & 2.28 \\
\hline Cerrato et al. 2005 & $0.520(0.306,0.884)$ & 2.48 \\
\hline Das et al. 2016 & $1.074(0.804,1.435)$ & 3.31 \\
\hline Kang and Lee. 2006 & $2.353(1.302,4.251)$ & 2.28 \\
\hline Slowik et al. 2003 & $0.693(0.254,1.894)$ & 1.29 \\
\hline Kessler et al. 1997 & $1.313(0.820,2.100)$ & 2.69 \\
\hline Wei et al. 2015 & $0.722(0.480,1.085)$ & 2.90 \\
\hline Chowdhury et al. 2001 & $1.182(0.660,2.118)$ & 2.31 \\
\hline MacLeod et al. 2001 & $0.695(0.454,1.064)$ & 2.84 \\
\hline Fayed et al. 2009 & $15.000(1.679,134.025)$ & 0.37 \\
\hline Catto et al. 2010 & $0.883(0.621,1.255)$ & 3.10 \\
\hline Margaglione et al. 1998 & $1.919(1.125,3.273)$ & 2.47 \\
\hline Kokubo et al. 2000 & $0.970(0.644,1.461)$ & 2.90 \\
\hline Saidi et al. 2009 & $3.096(2.048,4.681)$ & 2.88 \\
\hline Brewin et al. 2020 & $1.366(0.613,3.045)$ & 1.71 \\
\hline Gao et al. 2006 & $2.311(0.836,6.392)$ & 1.27 \\
\hline Souza et al. 2003 & $0.398(0.161,0.980)$ & 1.49 \\
\hline Hachinski et al. 1996 & $1.617(0.785,3.332)$ & 1.90 \\
\hline Couderc et al. 1993 & $0.692(0.340,1.409)$ & 1.93 \\
\hline Qian et al. 2012 & $1.475(0.558,3.902)$ & 1.35 \\
\hline Konialis et al. 2016 & $2.118(1.129,3.973)$ & 2.17 \\
\hline Morrison et al. 2002 & $1.227(0.939,1.604)$ & 3.37 \\
\hline Stankovic et al. 2004 & $2.013(1.059,3.826)$ & 2.13 \\
\hline Koopal et al. 2016 & $0.927(0.693,1.240)$ & 3.30 \\
\hline Fekih-Mrissa et al. 2014 & $13.129(0.672,256.598)$ & 0.21 \\
\hline Parfenov et al. 2007 & $1.381(0.723,2.637)$ & 2.12 \\
\hline Ji et al. 1998 & $2.491(1.232,5.037)$ & 1.95 \\
\hline Overall $(\mathrm{I}$-squared $=68.9 \%, \mathrm{p}=0.000)$ & $1.340(1.165,1.542)$ & 100.00 \\
\hline NOTE: Weights are from random effects analysis & & \\
\hline $\begin{array}{c}1 \\
.0039\end{array}$ & & \\
\hline
\end{tabular}

(b)

Figure 2: Continued. 


\begin{tabular}{|c|c|c|c|}
\hline \multicolumn{2}{|l|}{$\begin{array}{l}\text { Study } \\
\text { ID } \\
\end{array}$} & \multirow{2}{*}{$\frac{\mathrm{OR}(95 \% \mathrm{CI})}{2.789(1.076,7.228)}$} & \multirow{2}{*}{$\begin{array}{l}\% \\
\text { Weight } \\
3.07\end{array}$} \\
\hline Wu et al. 2020 & 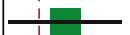 & & \\
\hline Zhao et al. 2017 & & $8.438(1.050,67.815)$ & 0.53 \\
\hline Coen Herak et al. 2017 & & $0.492(0.020,12.314)$ & 0.66 \\
\hline Das et al. 2016 & & $1.012(0.450,2.277)$ & 6.38 \\
\hline Atadzhanov et al. 2013 & & $0.213(0.011,3.999)$ & 1.71 \\
\hline Luo et al. 2015 & & $0.948(0.341,2.632)$ & 4.16 \\
\hline Wei et al. 2015 & & $4.164(0.851,20.376)$ & 0.97 \\
\hline Gelfand et al. 2013 & & $11.000(1.264,95.692)$ & 0.17 \\
\hline Parfenov et al. 2007 & & $1.841(0.325,10.432)$ & 1.09 \\
\hline Koopal et al. 2016 & & $0.890(0.429,1.849)$ & 8.77 \\
\hline Tamam et al. 2009 & & $0.500(0.030,8.331)$ & 0.71 \\
\hline Yan et al. 2015 & & $2.156(1.354,3.433)$ & 14.05 \\
\hline Balcerzyk et al. 2010 & & $3.923(0.424,36.305)$ & 0.53 \\
\hline Tascilar et al. 2009 & & $3.111(0.611,15.850)$ & 1.05 \\
\hline Wang et al. 2009 & & $4.849(1.325,17.754)$ & 1.35 \\
\hline Lai et al. 2007 & & $0.161(0.006,3.998)$ & 1.10 \\
\hline Chatzistefanidis et al. 2014 & & $2.449(0.445,13.494)$ & 0.98 \\
\hline Kang and Lee. 2006 & & $0.339(0.014,8.390)$ & 0.81 \\
\hline Baum et al. 2006 & +1 & $5.239(0.580,47.339)$ & 0.47 \\
\hline Pezzini et al. 2005 & & $3.301(0.133,81.891)$ & 0.26 \\
\hline Cerrato et al. 2005 & 1 & $0.687(0.169,2.787)$ & 2.55 \\
\hline Jin et al. 2004 & 단 & $1.539(0.254,9.342)$ & 1.07 \\
\hline Duzenli et al. 2004 & & $0.511(0.020,12.785)$ & 0.64 \\
\hline Slowik et al. 2003 & ta & $1.206(0.047,30.768)$ & 0.38 \\
\hline Souza et al. 2003 & & $2.390(0.096,59.531)$ & 0.30 \\
\hline Karttunen et al. 2002 & & $0.351(0.018,7.016)$ & 1.07 \\
\hline Morrison et al. 2002 & & $1.604(0.840,3.065)$ & 7.31 \\
\hline MacLeod et al. 2001 & & $0.782(0.155,3.939)$ & 1.81 \\
\hline Chowdhury et al. 2001 & & $1.319(0.183,9.504)$ & 0.93 \\
\hline Catto et al. 2000 & & $0.883(0.315,2.470)$ & 4.17 \\
\hline Kokubo et al. 2000 & & $0.457(0.059,3.518)$ & 2.03 \\
\hline Peng et al. 1999 & & $2.291(0.202,25.959)$ & 0.50 \\
\hline Ji et al. 1998 & & $8.409(0.428,165.235)$ & 0.24 \\
\hline Margaglione et al. 1998 & & $37.424(4.426,316.422)$ & 0.15 \\
\hline Frikke-Schmidt et al. 2001 & & $0.871(0.527,1.439)$ & 18.95 \\
\hline Saidi et al. 2009 & & $7.076(3.274,15.291)$ & 2.72 \\
\hline Hachinski et al. 1996 & & $3.638(0.366,36.140)$ & 0.48 \\
\hline Couderc et al. 1993 & 1 & $1.077(0.130,8.938)$ & 0.88 \\
\hline Qian et al. 2012 & $\mathrm{i}$ & $4.634(0.257,83.566)$ & 0.39 \\
\hline Konialis et al. 2016 & $\frac{1}{1}$ & $6.086(0.311,118.953)$ & 0.29 \\
\hline Fayed et al. 2009 & -1 & $22.913(1.196,438.832)$ & 0.18 \\
\hline Stankovic et al. 2004 & & $3.504(0.567,21.664)$ & 0.52 \\
\hline Pedro-Botet et al. 1992 & $1=$ & $2.556(0.611,10.689)$ & 1.35 \\
\hline Fekih-Mrissa et al. 2014 & & $37.000(1.004,1364.036)$ & 0.04 \\
\hline Brewin et al. 2020 & +1 & $4.157(1.307,13.220)$ & 1.21 \\
\hline Kessler et al. 1997 & & $3.951(0.807,19.351)$ & 1.00 \\
\hline Overall $(\mathrm{I}$-squared $=38.9 \%, \mathrm{p}=0.004)$ & \langle & $1.833(1.542,2.179)$ & 100.00 \\
\hline $\begin{array}{c}1 \\
.00073\end{array}$ & & 64 & \\
\hline
\end{tabular}

(c)

Figure 2: Continued. 


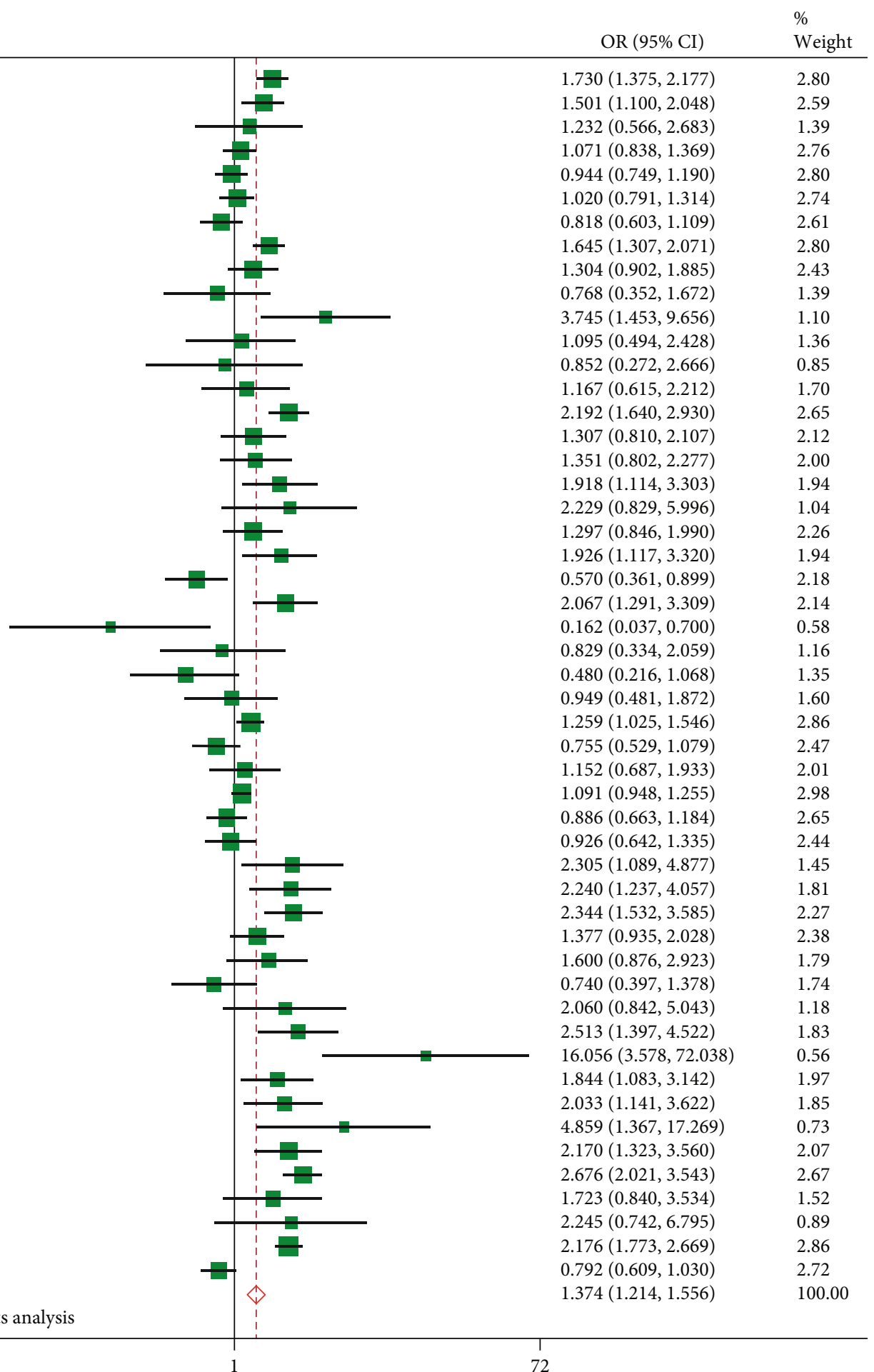

Wu et al. 2020

Zhao et al. 2017

Coen Herak et al. 2017

Das et al. 2016

Koopal et al. 2016

Luo et al. 2015

Wei et al. 2015

Yan et al. 2015

Chatzistefanidis et al. 2014

Atadzhanov et al. 2013

Gelfand et al. 2013

Balcerzyk et al. 2010

Tamam et al. 2009

Tascilar et al. 2009

Wang et al. 2009

Lai et al. 2007

Parfenov et al. 2007

Kang and Lee. 2006

Gao et al. 2006

Baum et al. 2006

Pezzini et al. 2005

Cerrato et al. 2005

Jin et al. 2004

Duzenli et al. 2004

Slowik et al. 2003

Souza et al. 2003

Karttunen et al. 2002

Morrison et al. 2002

MacLeod et al. 2001

Chowdhury et al. 2001

Frikke-Schmidt et al. 2001

Catto et al. 2000

Kokubo et al. 2000

Peng et al. 1999

Ji et al. 1998

Margaglione et al. 1998

Kessler et al. 1997

Hachinski et al. 1996

Couderc et al. 1993

Qain et al. 2012

Konialis et al. 2009

Fayed et al. 2009

Stankovic et al. 2004

Pedro-Botet et al. 1992

Fekih-Mrissa et al. 2014

Brewin et al. 2020

Saidi et al. 2009

Giassakis et al. 2007

Nakata et al. 1997

Szolnoki et al. 2002

Aalto-Setala et al. 1998

Overall (I-squared $=77.8 \%, \mathrm{p}=0.000$ )

NOTE: Weights are from random effects analysis

(d)

Figure 2: Continued. 


\begin{tabular}{|c|c|c|}
\hline $\begin{array}{l}\text { Study } \\
\text { ID }\end{array}$ & OR $(95 \% \mathrm{CI})$ & $\begin{array}{l}\% \\
\text { Weight }\end{array}$ \\
\hline Hachinski et al. 1996 & $1.584(0.811,3.092)$ & 1.81 \\
\hline Gelfand et al. 2013 & $3.500(1.057,11.586)$ & 0.91 \\
\hline Wu et al. 2020 & $1.823(1.426,2.331)$ & 2.93 \\
\hline Atadzhanov et al. 2013 & $0.914(0.371,2.253)$ & 1.33 \\
\hline Zhao et al. 2017 & $1.463(1.051,2.035)$ & 2.72 \\
\hline Balcerzyk et al. 2010 & $0.793(0.319,1.974)$ & 1.31 \\
\hline Coen Herak et al. 2017 & $1.331(0.584,3.033)$ & 1.47 \\
\hline Wang et al. 2009 & $2.503(1.828,3.429)$ & 2.76 \\
\hline Koopal et al. 2016 & $0.927(0.713,1.206)$ & 2.89 \\
\hline Yan et al. 2015 & $1.856(1.414,2.436)$ & 2.87 \\
\hline Wei et al. 2015 & $0.598(0.424,0.843)$ & 2.69 \\
\hline Peng et al. 1999 & $2.588(1.146,5.844)$ & 1.49 \\
\hline Chatzistefanidis et al. 2014 & $1.263(0.852,1.874)$ & 2.55 \\
\hline Gao et al. 2006 & $2.341(0.852,6.430)$ & 1.15 \\
\hline Tascilar et al. 2009 & $0.944(0.453,1.966)$ & 1.66 \\
\hline Tamam et al. 2009 & $0.912(0.252,3.303)$ & 0.82 \\
\hline Cerrato et al. 2005 & $0.525(0.320,0.862)$ & 2.26 \\
\hline Parfenov et al. 2007 & $1.509(0.835,2.730)$ & 2.00 \\
\hline Duzenli et al. 2004 & $0.167(0.038,0.736)$ & 0.66 \\
\hline Kang and Lee. 2006 & $2.171(1.221,3.859)$ & 2.04 \\
\hline Das et al. 2016 & $1.090(0.831,1.429)$ & 2.87 \\
\hline Jin et al. 2004 & $2.324(1.397,3.865)$ & 2.22 \\
\hline Baum et al. 2006 & $1.204(0.762,1.901)$ & 2.37 \\
\hline Souza et al. 2003 & $0.418(0.178,0.981)$ & 1.42 \\
\hline Pezzini et al. 2005 & $2.010(1.132,3.571)$ & 2.05 \\
\hline Slowik et al. 2003 & $0.737(0.274,1.982)$ & 1.18 \\
\hline Lai et al. 2007 & $1.489(0.886,2.501)$ & 2.20 \\
\hline Couderc et al. 1993 & $0.697(0.355,1.370)$ & 1.79 \\
\hline Karttunen et al. 2002 & $1.050(0.492,2.243)$ & 1.60 \\
\hline Morrison et al. 2002 & $1.293(1.019,1.641)$ & 2.95 \\
\hline MacLeod et al. 2001 & $0.701(0.473,1.041)$ & 2.54 \\
\hline Stankovic et al. 2004 & $2.129(1.169,3.875)$ & 1.98 \\
\hline Chowdhury et al. 2001 & $1.162(0.663,2.034)$ & 2.08 \\
\hline Ji et al. 1998 & $2.188(1.157,4.136)$ & 1.89 \\
\hline Frikke-Schmidt et al. 2001 & $1.152(0.982,1.351)$ & 3.11 \\
\hline Margaglione et al. 1998 & $2.093(1.287,3.405)$ & 2.29 \\
\hline Catto et al. 2000 & $0.879(0.636,1.214)$ & 2.74 \\
\hline Konialis et al. 2016 & $2.595(1.404,4.795)$ & 1.94 \\
\hline Kokubo et al. 2000 & $0.883(0.599,1.304)$ & 2.56 \\
\hline Pedro-Botet et al. 1992 & $2.144(1.107,4.152)$ & 1.83 \\
\hline Luo et al. 2015 & $1.024(0.780,1.344)$ & 2.87 \\
\hline Fayed et al. 2009 & $16.714(3.378,82.690)$ & 0.58 \\
\hline Kessler et al. 1997 & $1.282(0.836,1.966)$ & 2.45 \\
\hline Qian et al. 2012 & $1.759(0.684,4.525)$ & 1.25 \\
\hline Brewin et al. 2020 & $2.307(1.218,4.368)$ & 1.88 \\
\hline Fekih-Mrissa et al. 2014 & $20.879(1.102,395.483)$ & 0.20 \\
\hline Li et al. 2016 & $1.739(0.995,3.038)$ & 2.09 \\
\hline Saidi et al. 2009 & $3.123(2.195,4.444)$ & 2.66 \\
\hline Artieda et al. 2008 & $1.282(0.730,2.253)$ & 2.07 \\
\hline Schneider et al. 2005 & $2.107(1.157,3.837)$ & 1.98 \\
\hline Overall $(\mathrm{I}$-squared $=74.9 \%, \mathrm{p}=0.000)$ & $1.377(1.203,1.576)$ & 100.00 \\
\hline NOTE: Weights are from random effects analysis & & \\
\hline
\end{tabular}

(e)

Figure 2: Continued. 


\begin{tabular}{|c|c|c|c|}
\hline $\begin{array}{l}\text { Study } \\
\text { ID }\end{array}$ & & OR $(95 \% \mathrm{CI})$ & $\begin{array}{l}\% \\
\text { Weight }\end{array}$ \\
\hline Catto et al. 2000 & & $1.458(0.348,6.112)$ & 2.88 \\
\hline Peng et al. 1999 & & $2.000(0.051,78.250)$ & 0.37 \\
\hline Wu et al. 2020 & & $1.806(0.552,5.908)$ & 3.86 \\
\hline Zhao et al. 2017 & & $9143(0.905,92.398)$ & 0.54 \\
\hline Coen Herak et al. 2017 & & $0.067(0.001,5.494)$ & 1.39 \\
\hline Das et al. 2016 & 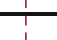 & $0.667(0.149,2.979)$ & 3.93 \\
\hline Wei et al. 2015 & & $4.109(0.776,21.760)$ & 1.45 \\
\hline Koopal et al. 2016 & & $0.706(0.256,1.946)$ & 8.15 \\
\hline Wang et al. 2009 & & $2.506(0.658,9.540)$ & 2.91 \\
\hline Luo et al. 2015 & -1 & $0.538(0.141,2.063)$ & 5.37 \\
\hline Yan et al. 2015 & & $3.175(1.701,5.924)$ & 10.27 \\
\hline Atadzhanov et al. 2013 & & $0.113(0.005,2.539)$ & 2.94 \\
\hline Chatzistefanidis et al. 2014 & & $5.333(0.618,45.991)$ & 0.66 \\
\hline Gelfand et al. 2013 & & $1.500(0.106,21.312)$ & 0.83 \\
\hline Tamam et al. 2009 & & $0.500(0.013,19.562)$ & 0.74 \\
\hline Tascilar et al. 2009 & & $8.167(1.027,64.936)$ & 0.59 \\
\hline Lai et al. 2007 & & $0.175(0.006,5.041)$ & 1.63 \\
\hline Parfenov et al. 2007 & & $6.000(0.354,101.568)$ & 0.37 \\
\hline Baum et al. 2006 & & $4.000(0.340,47.112)$ & 0.66 \\
\hline Pezzini et al. 2005 & & $3000(0.060,151.190)$ & 0.28 \\
\hline Cerrato et al. 2005 & & $3.000(0.095,95.170)$ & 0.38 \\
\hline Jin et al. 2004 & & $1.000(0.080,12.557)$ & 1.11 \\
\hline Duzenli et al. 2004 & & $0.556(0.013,24.513)$ & 0.70 \\
\hline Souza et al. 2003 & & $15.000(0.182,1236.183)$ & 0.09 \\
\hline Karttunen et al. 2002 & & $0.143(0.003,5.946)$ & 1.39 \\
\hline Morrison et al. 2002 & & $1.100(0.478,2.529)$ & 9.78 \\
\hline Kessler et al. 1997 & & $4.200(0.586,30.095)$ & 0.93 \\
\hline MacLeod et al. 2001 & & $0.857(0.124,5.944)$ & 2.05 \\
\hline Saidi et al. 2009 & & $3.136(1.273,7.723)$ & 5.10 \\
\hline Frikke-Schmidt et al. 2001 & & $0.712(0.371,1.366)$ & 20.07 \\
\hline Kokubo et al. 2000 & & $0.308(0.024,3.968)$ & 2.01 \\
\hline Ji et al. 1998 & & $9.000(0.340,238.210)$ & 0.27 \\
\hline Margaglione et al. 1998 & & $65.000(2.239,1887.351)$ & 0.09 \\
\hline Hachinski et al. 1996 & & $3.000(0.084,107.447)$ & 0.31 \\
\hline Couderc et al. 1993 & 1 & $2.200(0.075,64.904)$ & 0.46 \\
\hline Fayed et al. 2009 & 1 & $3.400(0.120,96.700)$ & 0.39 \\
\hline Stankovic et al. 2004 & 1 & $10.714(0.399,287.828)$ & 0.23 \\
\hline Pedro-Botet et al. 1992 & - & $9.286(0.342,252.450)$ & 0.25 \\
\hline Brewin et al. 2020 & 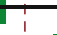 & $0.825(0.211,3.219)$ & 4.25 \\
\hline Chowdhury et al. 2001 & & $3.000(0.078,115.338)$ & 0.33 \\
\hline Overall (I-squared $=23.8 \%, p=0.092)$ & $\diamond$ & $1.625(1.281,2.060)$ & 100.00 \\
\hline
\end{tabular}

(f)

Figure 2: Continued. 


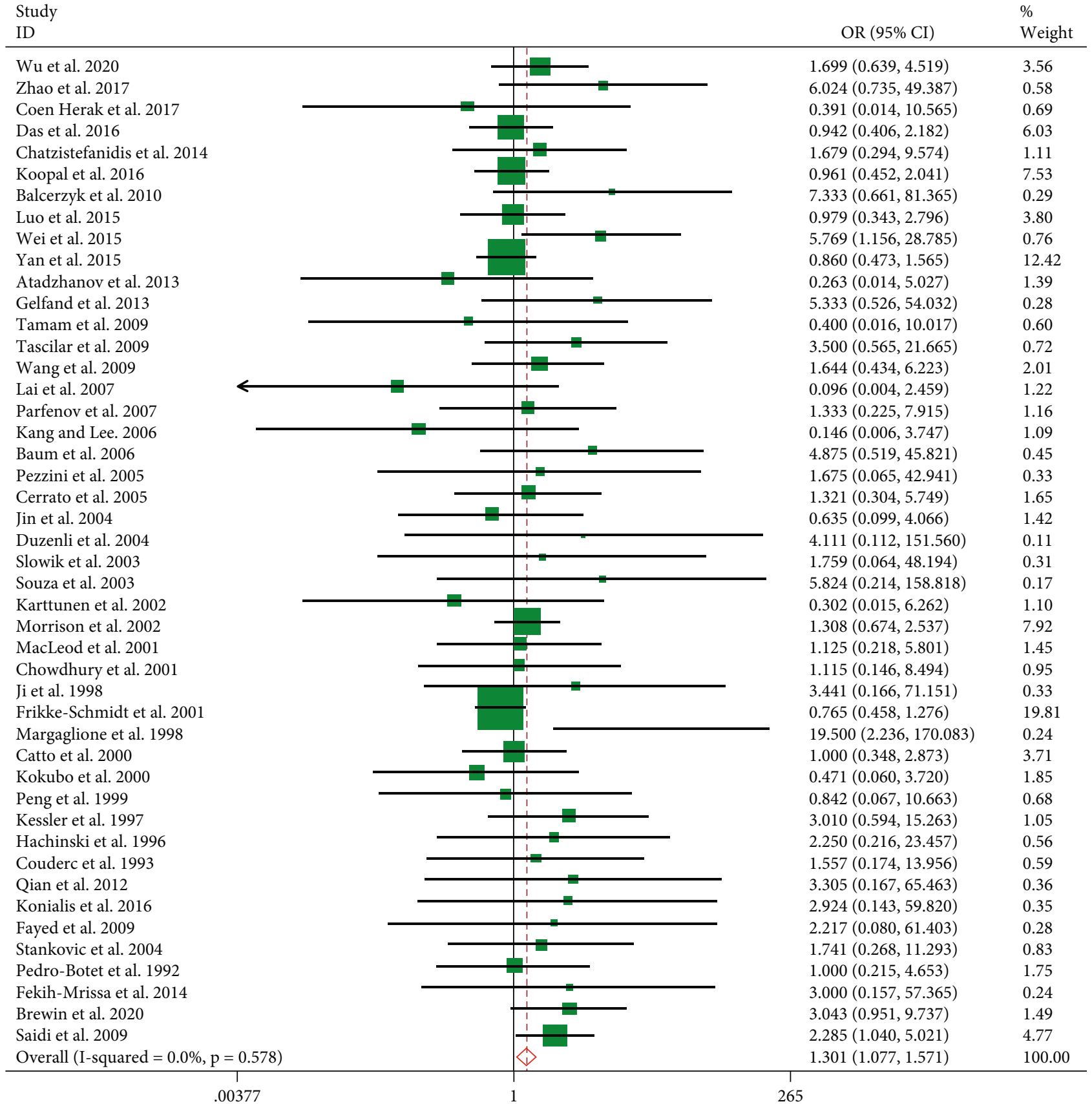

(g)

Figure 2: (a-g) Forest plots of the relationships between APOE gene polymorphisms in all studies included. (a) Forest plot of $\varepsilon 2 / \varepsilon 4$ vs. $\varepsilon 3 / \varepsilon 3$ comparison. (b) Forest plot of $\varepsilon 3 / \varepsilon 4$ vs. $\varepsilon 3 / \varepsilon 3$ comparison. (c) Forest plot of APOE $\varepsilon 4 / \varepsilon 4$ vs. the $\varepsilon 3 / \varepsilon 3$ genotype. (d) Forest plot of the APOE $\varepsilon 4$ allele vs. $\varepsilon 3$ allele. (e) Forest plot of APOE $\varepsilon 4$ carriers vs. non- $\varepsilon 4$ carriers. (f) Forest plot of APOE $\varepsilon 4 / \varepsilon 4$ vs. $\varepsilon 2 / \varepsilon 4$. (g) Forest plot of APOE $\varepsilon 4 / \varepsilon 4$ vs. $\varepsilon 3 / \varepsilon 4$.

3.2.4. APOE $\varepsilon 2$ Carrier Comparisons. In the genetic model of $\varepsilon 2$ carriers vs. non- $\varepsilon 2$ carriers, there was no association with the IS risk (pooled OR $=0.956,95 \%$ CI 0.841-1.086, $P=0.49$ ) (Table 2).

3.2.5. APOE \&4 Homozygosis versus APOE $\varepsilon 4$ Heterozygote Comparisons. Given the above, the APOE $\varepsilon 4$ mutation was linked to IS risk. To identify whether there is a dose-response relationship between the $\varepsilon 4$ allele and IS or not, we implemented the comparisons between the $\varepsilon 4 /$ $\varepsilon 4$ genotype and $\varepsilon 4$ heterozygotes $(\varepsilon 2 / \varepsilon 4$ or $\varepsilon 3 / \varepsilon 4$ genotype). Compared with the $\varepsilon 2 / \varepsilon 4$ and $\varepsilon 3 / \varepsilon 4$ genotypes, the IS risk ORs for $\varepsilon 4 / \varepsilon 4$ genotypes were 1.625 (95\% CI, 1.281-2.060, $P<0.0001$ ) and 1.301 (95\% CI, 1.077-1.571, 


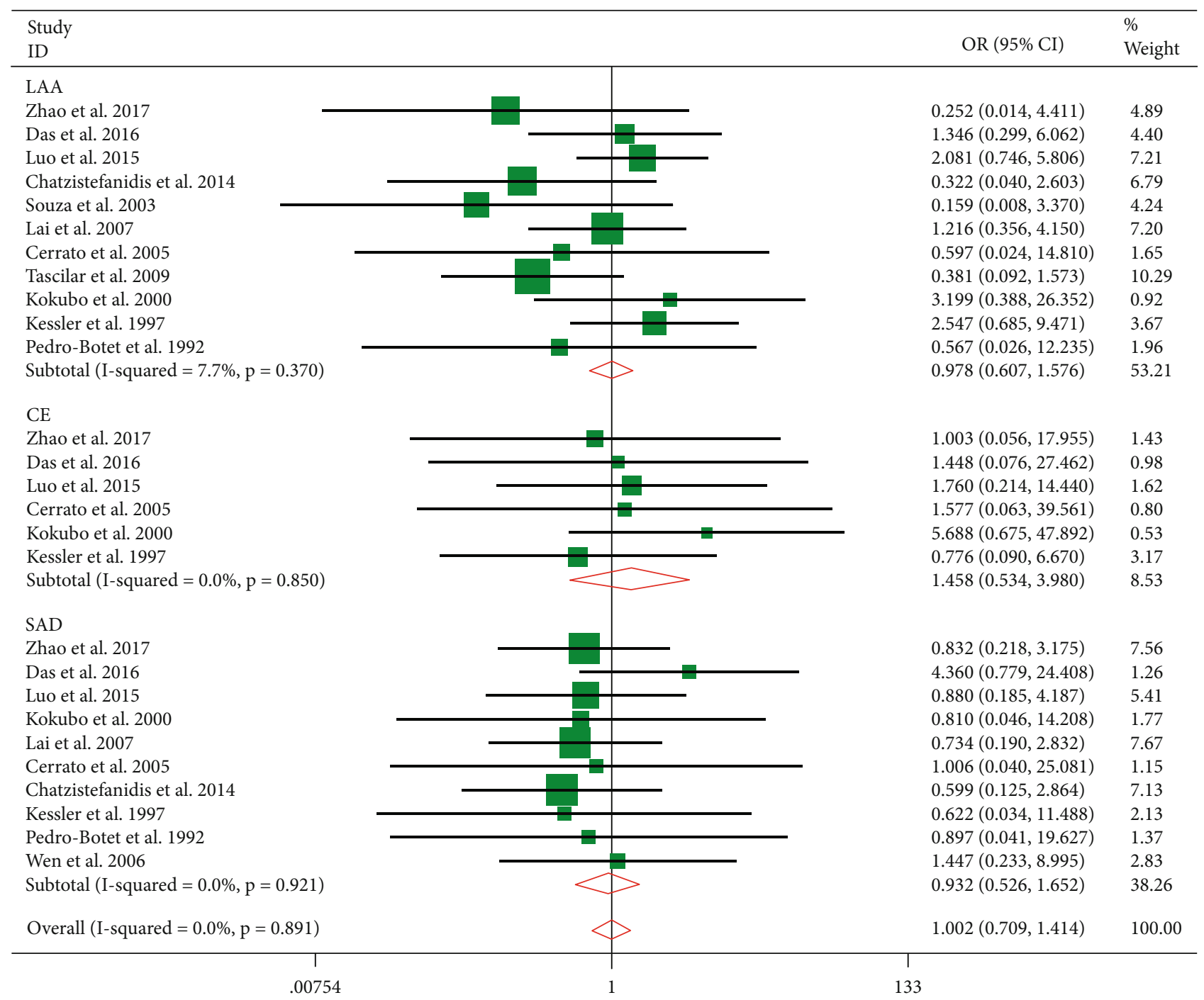

(a)

FIgURe 3: Continued. 


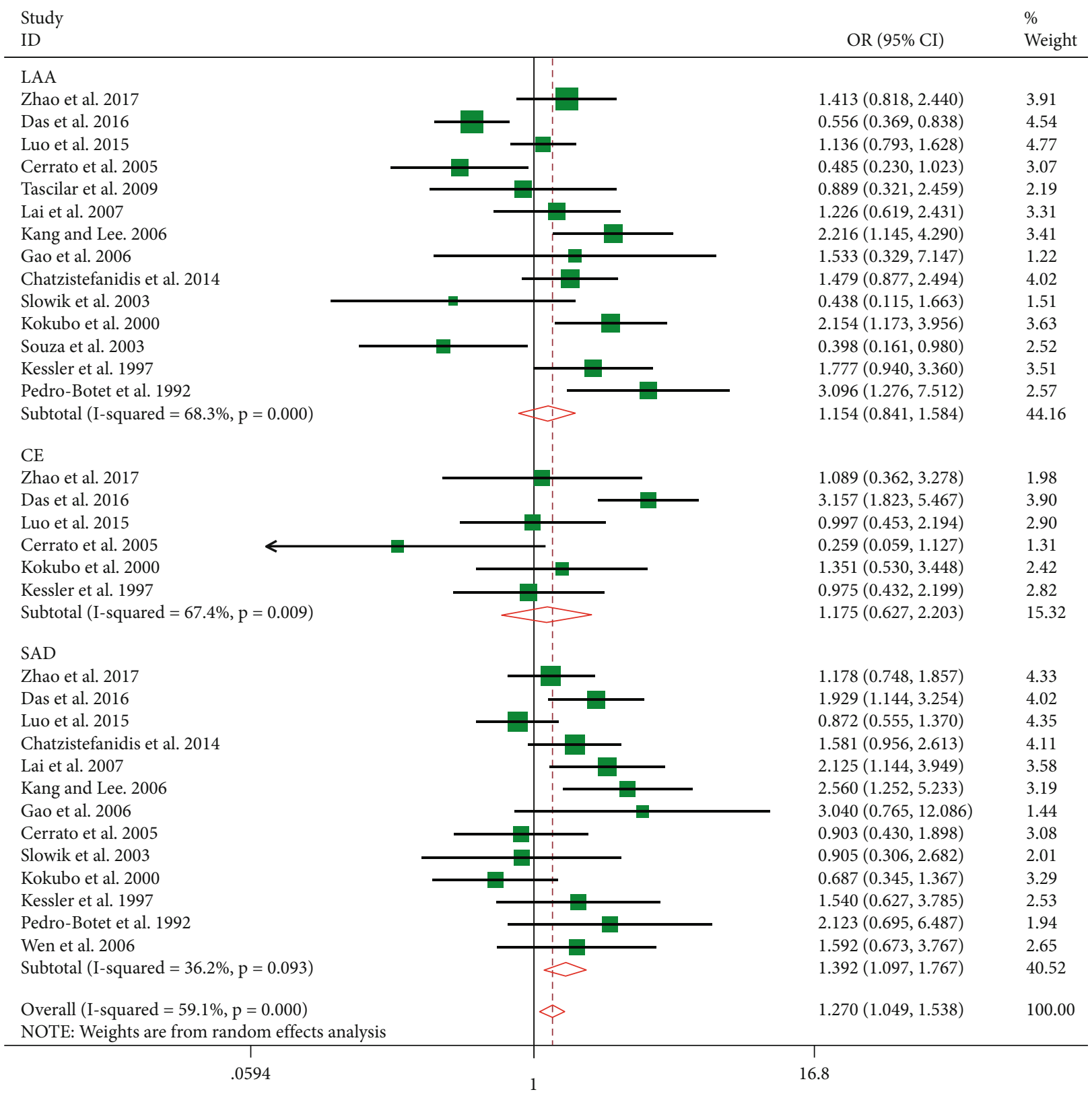

(b)

Figure 3: Continued. 


\begin{tabular}{|c|c|c|}
\hline $\begin{array}{l}\text { Study } \\
\text { ID }\end{array}$ & OR (95\% CI) & $\begin{array}{l}\% \\
\text { Weight }\end{array}$ \\
\hline LAA & & \\
\hline Das et al. 2016 & $0.748(0.260,2.147)$ & 17.48 \\
\hline Zhao et al. 2017 & $12.918(1.327,125.717)$ & 0.78 \\
\hline Luo et al. 2015 & $1.189(0.354,3.988)$ & 9.73 \\
\hline Chatzistefanidis et al. 2014 & $2.574(0.358,18.504)$ & 2.31 \\
\hline Tascilar et al. 2009 & $3.111(0.611,15.850)$ & 4.00 \\
\hline Lai et al. 2007 & $0.338(0.014,8.416)$ & 3.07 \\
\hline Kang and Lee. 2006 & $0.560(0.023,13.917)$ & 2.31 \\
\hline Cerrato et al. 2005 & $0.449(0.049,4.078)$ & 5.85 \\
\hline Slowik et al. 2003 & $2.633(0.102,68.073)$ & 1.07 \\
\hline Souza et al. 2003 & $2.390(0.096,59.531)$ & 1.15 \\
\hline Kokubo et al. 2000 & $0.934(0.054,16.054)$ & 2.11 \\
\hline Kessler et al. 1997 & $1.910(0.169,21.616)$ & 1.70 \\
\hline Pedro-Botet et al. 1992 & $1.917(0.302,12.171)$ & 3.07 \\
\hline Subtotal $(\mathrm{I}$-squared $=0.0 \%, \mathrm{p}=0.706)$ & $1.367(0.836,2.236)$ & 54.63 \\
\hline $\mathrm{CE}$ & & \\
\hline Zhao et al. 2017 & $5.682(0.225,143.647)$ & 0.35 \\
\hline Das et al. 2016 & $2.202(0.473,10.253)$ & 3.42 \\
\hline Luo et al. 2015 & $0.818(0.046,14.443)$ & 2.34 \\
\hline Cerrato et al. 2005 & $0.526(0.028,9.998)$ & 3.19 \\
\hline Kokubo et al. 2000 & $1.641(0.094,28.649)$ & 1.22 \\
\hline Kessler et al. 1997 & $2.328(0.205,26.462)$ & 1.45 \\
\hline Subtotal $(\mathrm{I}$-squared $=0.0 \%, \mathrm{p}=0.896)$ & $1.543(0.591,4.029)$ & 11.98 \\
\hline SAD & & \\
\hline Das et al. 2016 & $0.727(0.093,5.706)$ & 5.02 \\
\hline Zhao et al. 2017 & $11.091(1.286,95.680)$ & 1.28 \\
\hline Luo et al. 2015 & $0.880(0.185,4.187)$ & 7.28 \\
\hline Chatzistefanidis et al. 2014 & $2.397(0.334,17.218)$ & 2.43 \\
\hline Lai et al. 2007 & $0.306(0.012,7.623)$ & 3.22 \\
\hline Kang and Lee. 2006 & $0.848(0.034,21.167)$ & 1.75 \\
\hline Cerrato et al. 2005 & $0.335(0.018,6.335)$ & 4.56 \\
\hline Kokubo et al. 2000 & $1.068(0.137,8.303)$ & 3.59 \\
\hline Kessler et al. 1997 & $8.278(1.098,62.405)$ & 0.88 \\
\hline Pedro-Botet et al. 1992 & $4.600(0.845,25.052)$ & 2.09 \\
\hline Wen et al. 2006 & $4.341(0.383,49.255)$ & 1.29 \\
\hline Subtotal (I-squared $=10.0 \%, \mathrm{p}=0.349)$ & $1.809(1.030,3.175)$ & 33.39 \\
\hline Overall (I-squared $=0.0 \%, p=0.802)$ & $1.536(1.086,2.171)$ & 100.00 \\
\hline
\end{tabular}

(c)

FIgURE 3: Continued. 


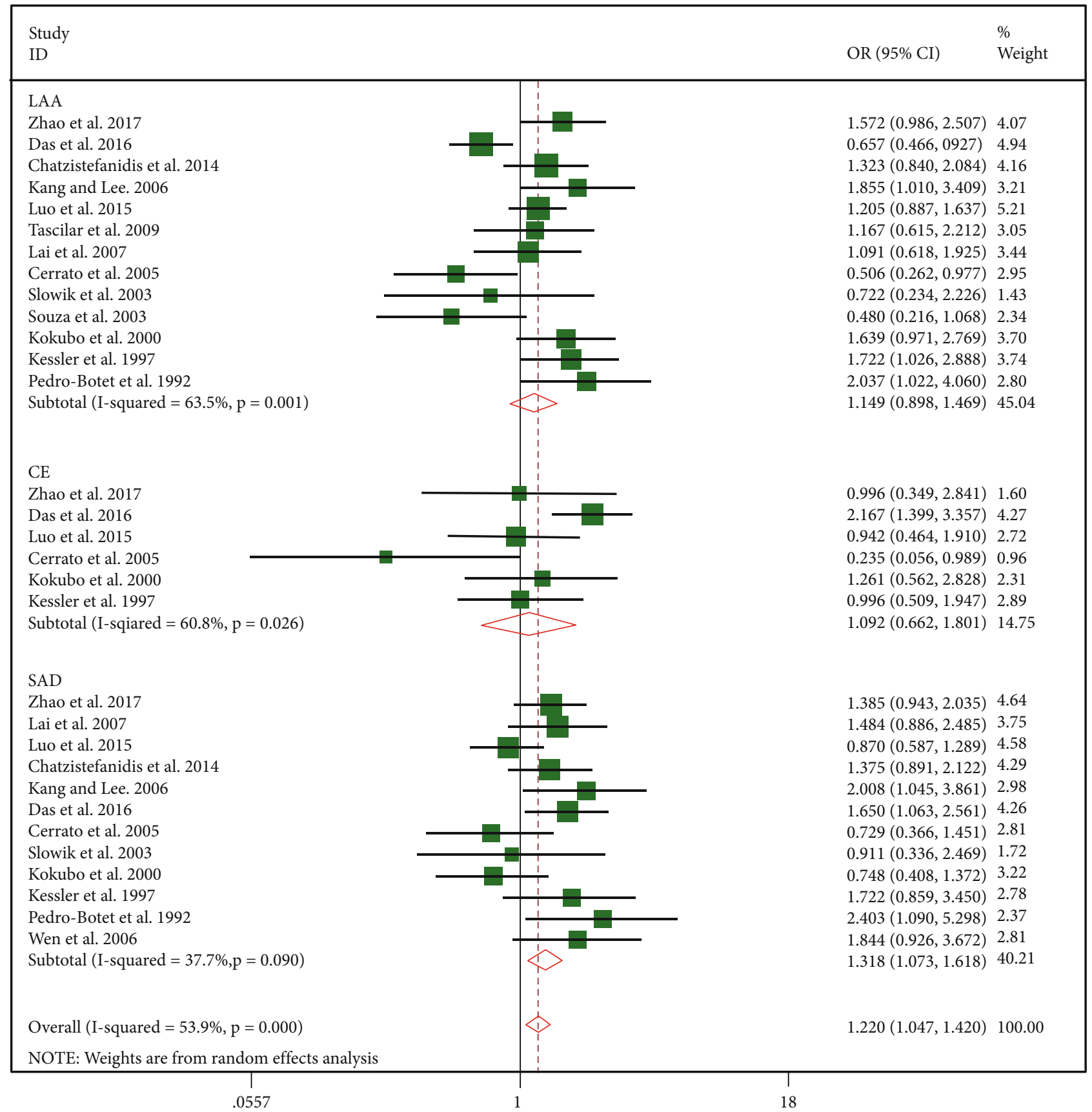

(d)

FIgURE 3: Continued. 


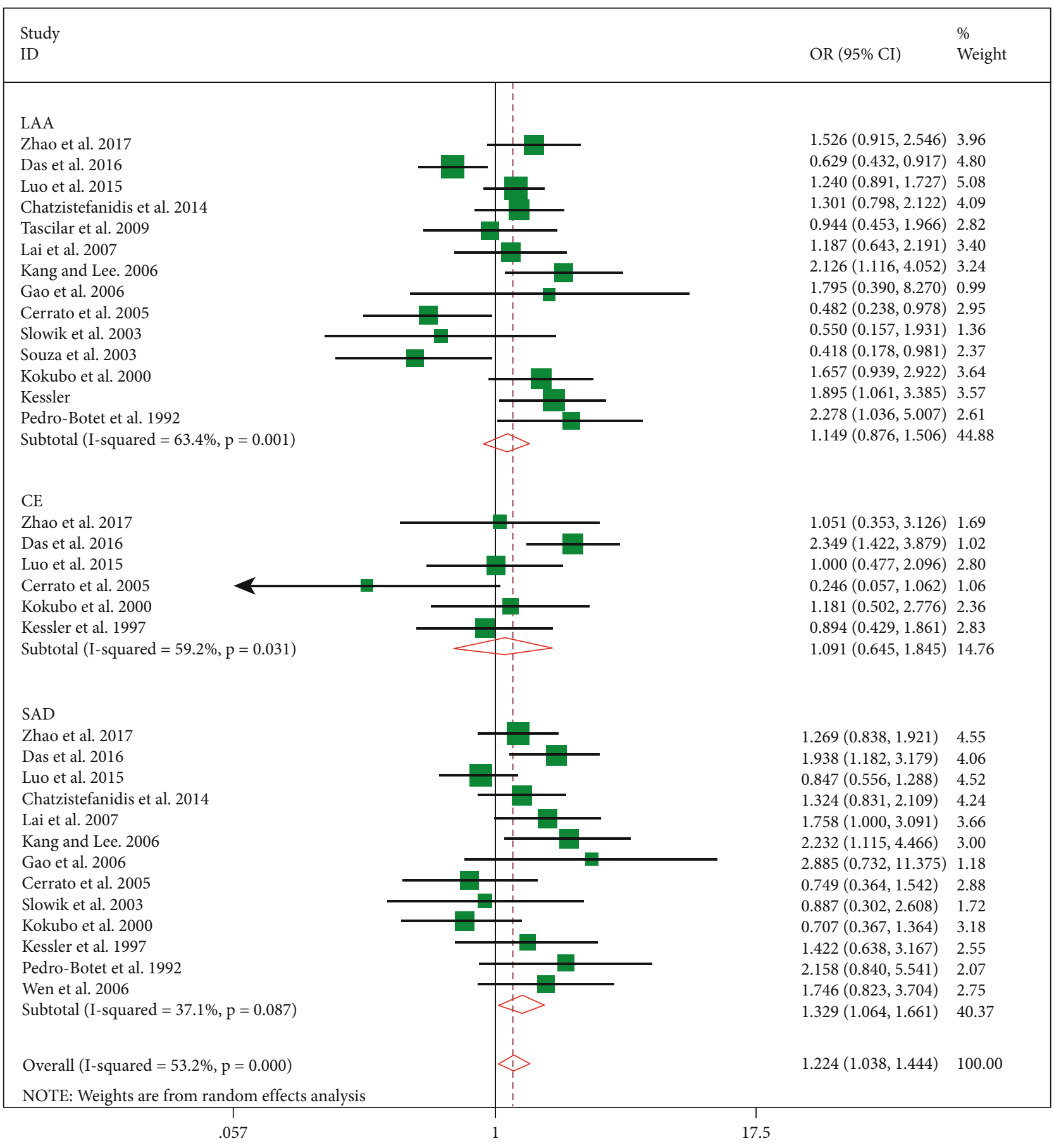

(e)

Figure 3: Continued. 


\begin{tabular}{|c|c|c|c|}
\hline \multicolumn{2}{|l|}{$\begin{array}{l}\text { Study } \\
\text { ID }\end{array}$} & \multirow[t]{2}{*}{ OR $(95 \% \mathrm{CI})$} & $\begin{array}{l}\% \\
\text { Weight }\end{array}$ \\
\hline LAA & 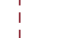 & & \\
\hline Zhao et al. 2017 & $\frac{1}{4}$ & $39.667(1.279,1229.867)$ & 0.42 \\
\hline Das et al. 2016 & 1 & $0.556(0.090,3.445)$ & 11.81 \\
\hline Tascilar et al. 2009 & $\frac{1}{1}$ & $8.167(1.027,64.936)$ & 2.49 \\
\hline Luo et al. 2015 & i & $0.571(0.119,2.751)$ & 16.33 \\
\hline Chatzistefanidis et al. 2014 & 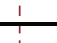 & $8.000(0.459,139.290)$ & 1.21 \\
\hline Lai et al. 2007 & 1 & $0.282(0.009,8.418)$ & 5.48 \\
\hline Cerrato et al. 2005 & $\frac{1}{1}$ & $1.000(0.025,40.276)$ & 2.21 \\
\hline Souza et al. 2003 & $\frac{1}{1}$ & $15.000(0.182,1236.183)$ & 0.39 \\
\hline Kokubo et al. 2000 & $\frac{1}{1}$ & $0.210(0.008,5.769)$ & 6.64 \\
\hline Kessler et al. 1997 & $\frac{1}{1}$ & $0.750(0.050,11.311)$ & 4.84 \\
\hline Pedro-Botet et al. 1992 & & $3.571(0.114,111.707)$ & 1.53 \\
\hline Subtotal $(\mathrm{I}$-squared $=27.9 \%, \mathrm{p}=0.179)$ & & $1.551(0.791,3.043)$ & 53.36 \\
\hline SAD & & & \\
\hline Zhao et al. 2017 & & $13.333(1.069,166.374)$ & 1.39 \\
\hline Das et al. 2016 & $\frac{1}{1}$ & $0.167(0.012,2.368)$ & 9.94 \\
\hline Luo et al. 2015 & + & $1.000(0.112,8.947)$ & 6.30 \\
\hline Chatzistefanidis et al. 2014 & $\frac{1}{1}$ & $4.000(0.329,48.656)$ & 2.25 \\
\hline Lai et al. 2007 & - & $0.407(0.013,12.636)$ & 4.43 \\
\hline Kessler et al. 1997 & -1 & $13.000(0.448,377.470)$ & 0.82 \\
\hline Kokubo et al. 2000 & + & $1.889(0.069,51.917)$ & 2.21 \\
\hline Pedro-Botet et al. 1992 & & $5.000(0.170,146.642)$ & 1.38 \\
\hline Wen et al. 2006 & & $3.000(0.150,59.890)$ & 1.97 \\
\hline Subtotal $(\mathrm{I}$-squared $=6.7 \%, \mathrm{p}=0.380)$ & & $2.115(0.919,4.867)$ & 30.69 \\
\hline $\mathrm{CE}$ & & & \\
\hline Das et al. 2016 & & $1.800(0.072,45.135)$ & 2.46 \\
\hline Luo et al. 2015 & & $0.333(0.012,9.395)$ & 5.28 \\
\hline Kokubo et al. 2000 & i & $0.210(0.008,5.769)$ & 6.64 \\
\hline Kessler et al. 1997 & +1 & $3.000(0.122,73.642)$ & 1.57 \\
\hline Subtotal $(\mathrm{I}$-squared $=0.0 \%, \mathrm{p}=0.616)$ & $\frac{1}{1}$ & $0.771(0.177,3.352)$ & 15.96 \\
\hline Overall $(\mathrm{I}$-squared $=8.9 \%, \mathrm{p}=0.338)$ & $\frac{1}{4}$ & $1.600(0.980,2.610)$ & 100.00 \\
\hline
\end{tabular}

(f)

Figure 3: Continued. 


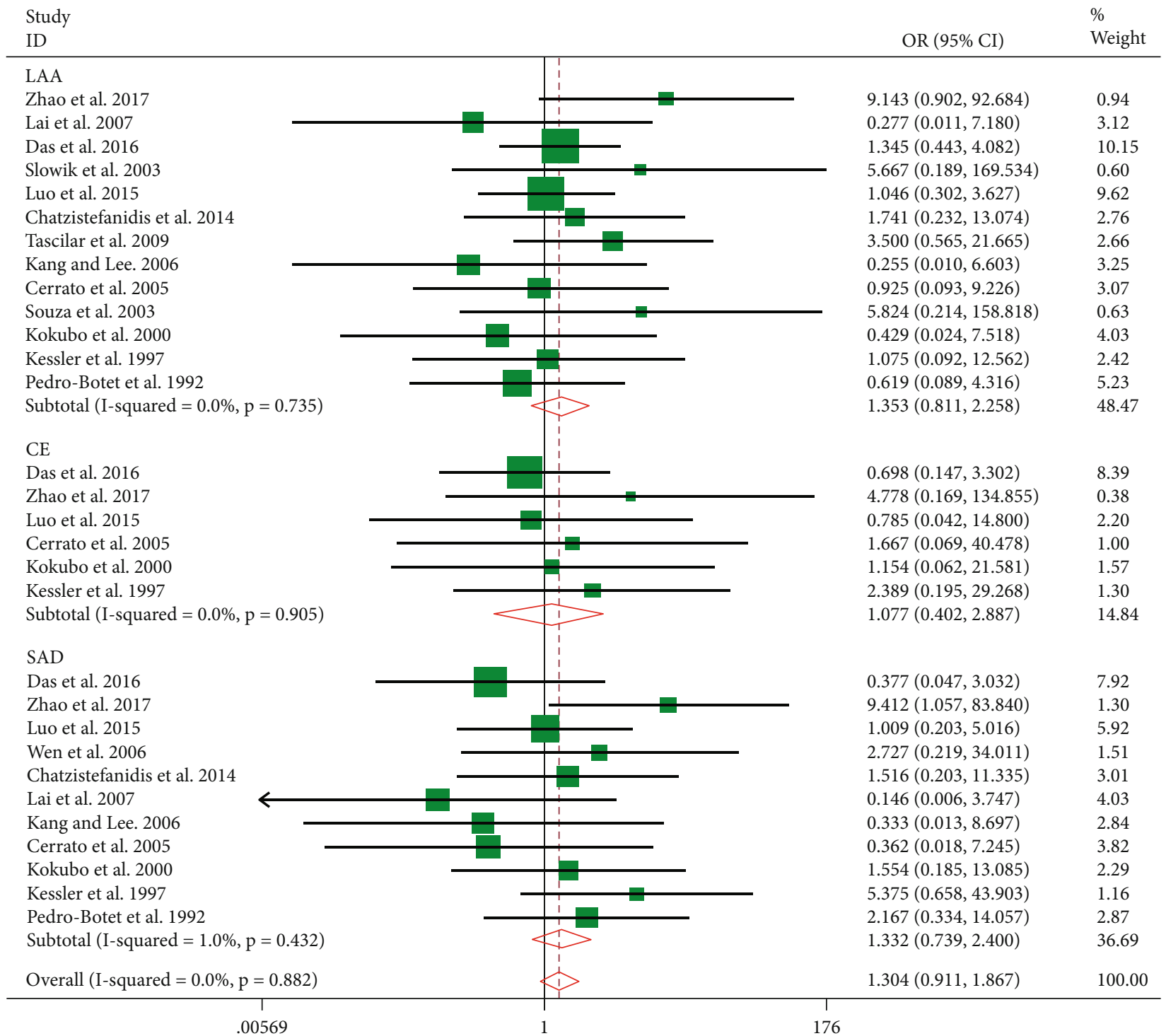

$(\mathrm{g})$

FIGURE 3: (a-g) Forest plots of the relationships between APOE gene polymorphisms in subgroup analysis. (a) Forest plot of $\varepsilon 2 / \varepsilon 4$ vs. $\varepsilon 3 / \varepsilon 3$ comparison. (b) Forest plot of $\varepsilon 3 / \varepsilon 4$ vs. $\varepsilon 3 / \varepsilon 3$ comparison. (c) Forest plot of APOE $\varepsilon 4 / \varepsilon 4$ vs. the $\varepsilon 3 / \varepsilon 3$ genotype. (d) Forest plot of the APOE $\varepsilon 4$ allele vs. $\varepsilon 3$ allele. (e) Forest plot of APOE $\varepsilon 4$ carriers vs. non- $\varepsilon 4$ carriers. (f) Forest plot of APOE $\varepsilon 4 / \varepsilon 4$ vs. $\varepsilon 2 / \varepsilon 4$. (g) Forest plot of APOE $\varepsilon 4 / \varepsilon 4$ vs. $\varepsilon 3 / \varepsilon 4$.

$P=0.01$ ), respectively (Figures $2(\mathrm{f})$ and $2(\mathrm{~g})$ ); this part provided evidence that $\varepsilon 4$ homozygosis might generate a higher risk of IS than $\varepsilon 4$ heterozygotes.

\subsection{Main Results of the Relationship between APOE Gene} and Three IS Subtypes. We further investigated on the correlation of APOE gene polymorphisms and risks of IS subtypes by making comparisons in five genetic models, with a particular focus on the APOE $\varepsilon 4$ mutation. Subgroup analyses showed that APOE $\varepsilon 4$ mutation significantly increased $\mathrm{SAD}$ risk ( $\varepsilon 4$ allele vs. $\varepsilon 3$ allele: pooled $\mathrm{OR}=1.318,95 \%$

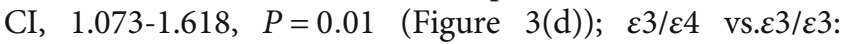
pooled $\mathrm{OR}=1.392, \quad 95 \% \quad \mathrm{CI}, \quad 1.097-1.767, \quad P=0.01$ (Figure $3(\mathrm{~b})$ ); $\varepsilon 4 / \varepsilon 4$ vs. $\varepsilon 3 / \varepsilon 3$ : pooled $\mathrm{OR}=1.809$, 95\%, CI $1.030-3.175, P=0.04$ (Figure $3(\mathrm{c})$ ); and APOE $\varepsilon 4$ carriers vs. non-APOE $\varepsilon 4$ carriers: pooled $\mathrm{OR}=1.329,95 \% \mathrm{CI}$, 1.064-1.661, $P=0.01$ (Figure 3(e))). But genotype $\varepsilon 2 / \varepsilon 4 \mathrm{did}$ not increase the risk of SAD onset (Figure $3(\mathrm{a})$ ). The result of APOE $\varepsilon 4$ homozygosis versus $\varepsilon 4$ heterozygote comparisons $(\varepsilon 4 / \varepsilon 4$ vs. $\varepsilon 2 / \varepsilon 4$ and $\varepsilon 4 / \varepsilon 4$ vs. $\varepsilon 3 / \varepsilon 4)$ was a matter of concern: APOE $\varepsilon 4$ mutation could not cause a cumulative effect in generating higher risk of SAD onset, as showed in Figures 3(f) and 3(g).

3.4. Sensitivity Analysis. Sensitivity analysis was performed by removing studies one by one to check the effect of the individual study on overall ORs. No single study influenced on the pooled ORs and 95\% CIs in all genetic model comparisons as our data showed (supplementary material table 4). 


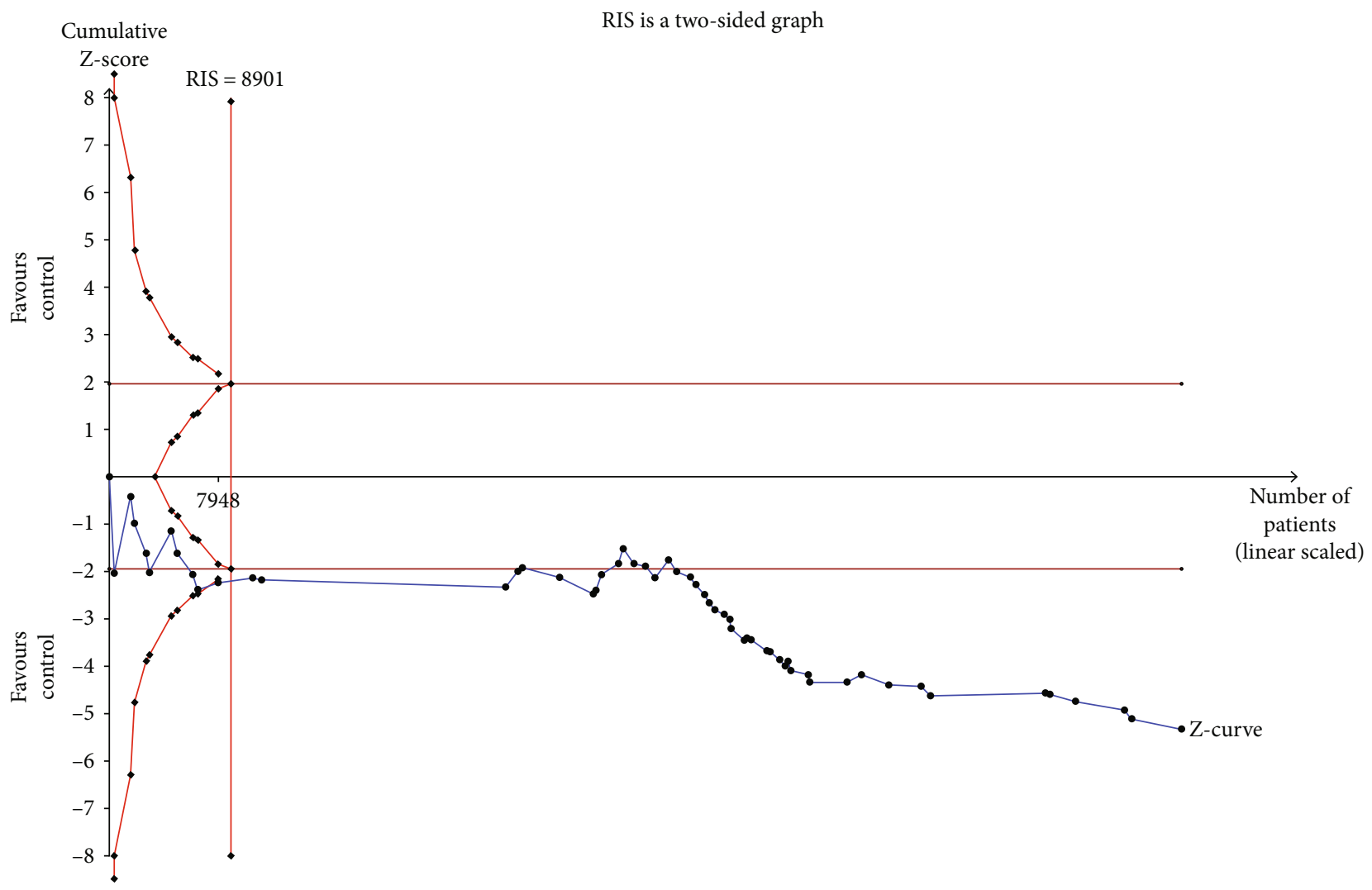

Figure 4: Trial sequential analysis of the association between APOE gene polymorphisms and ischemic stroke.

3.5. Publication Bias. We carried out publication bias analysis by using funnel plots as qualitative description and Egger's regression tests as quantitative outcome. Funnel plots of all genetic model comparisons did not exhibit apparent asymmetry (several funnel plots were showed in supplementary material figure 1 and 2 ). In addition to subtype analysis of $\varepsilon 2 / \varepsilon 2$ vs. $\varepsilon 3 / 3$, all the Egger's regression test outcomes indicated that there existed no evident publication bias with all $P$ values exceeding 0.1 (supplementary material table 5). The above results showed that publication bias of our metaanalysis was not significant.

3.6. Regression Analysis. Meta-regression analysis was then performed to explore sources of heterogeneity as shown in supplementary material table 5, considering the year of publication, region, sample size, genotyping method, HWE, NOS score, and source of control. However, the $P$ value of each factor affecting overall heterogeneity was not statistically significant in comparisons of $\varepsilon 3 / \varepsilon 4$ vs. $\varepsilon 3 / 3, \varepsilon 4$ vs. non- $\varepsilon 4, \varepsilon 2$ vs. non- $\varepsilon 2$, $\varepsilon 4$ allele vs. $\varepsilon 3$ allele, and $\varepsilon$ allele vs. $\varepsilon 3$ allele (supplementary material figure 3 ). Heterogeneity sources were unascertainable.

3.7. The Result of Trial Sequential Analysis (TSA). The RIS was 8901 samples and the sample size of our meta-analysis reached it. Moreover, the cumulative $z$-curve crossed the trial sequential monitoring boundary before reaching the RIS as showed in Figure 4. The result of TSA guaranteed the stability of our meta-analysis results. Our sample size was proved to be enough for evaluating the relationship between APOE polymorphisms and IS risk.

\section{Discussion}

Recently, scholars explored more how gene polymorphisms were contributing to the occurrence and prognosis of diseases. And several previous publications had well explored how gene polymorphisms related to diseases onset and potential mechanisms $[74,75]$. As a heterogeneous multifactorial disorder, ischemic stroke could be regulated by certain gene synthesis and specific gene products. The genes involved in the pathological process of stroke are also worth of attention. Apolipoprotein $\mathrm{E}$ has been proven to affect atherosclerosis, neurodegeneration, and the process of nerve damage repair. That is why we explored the relationship between APOE gene polymorphisms and ischemic stroke risk.

APOE is a 299-amino acid protein encoded by the APOE gene of three common polymorphisms, $\varepsilon 2$, $\varepsilon 3$, and $\varepsilon 4$. The correlation of APOE gene polymorphisms and the risk of cerebral vascular and degenerative diseases have been investigated a lot, especially in Alzheimer's disease (AD) and cerebral amyloid angiopathy (CAA) [76]. APOE $\varepsilon 4$ is associated with increased risk for AD whereas APOE $\varepsilon 2$ is associated with decreased risk [77]. Mirza et al. performed a metaanalysis to find that greater $\mathrm{WMH}$ volume was associated with worse performance on all cognitive domains in APOE $\varepsilon 4$ carriers only in $\mathrm{AD}$ [78]. Charidimou et al. proved that 
the APOE $\varepsilon 2$ allele might be associated with the pathophysiology and severity of cortical superficial siderosis in CAA [79]. As to IS, there existed quite many researches with inconsistent conclusions. Besides method differences, ethnic difference and unclarified pathophysiological mechanisms are probable reasons of the inconsistency.

In a meta-analysis in 1999, McCarron et al. found that the $\varepsilon 4$ allele and carriers were more frequent among patients with ischemic cerebrovascular disease, compared with control subjects $(27 \%$ versus $18 \%$; odds ratio, $1.73 ; 95 \% \mathrm{CI}$, 1.34-2.23; $P<0.0001$ ) [13]. In another meta-analysis based on Chinese population, the $\varepsilon 4$ allele is associated with an increased risk of developing cerebral infarction, in which the adjusted risk estimate for the $\varepsilon 4$ allele versus $\varepsilon 3$ allele was significant $(\mathrm{OR}=2.00,95 \%$ CI $1.59-2.53, P<0.0001)$ [14]. Our estimates seemed to be coinciding with the above ones. Compared with the $\varepsilon 3$ allele, the $\varepsilon 4$ allele showed a higher risk of IS. Compared with $\varepsilon 3 / \varepsilon 3$, both $\varepsilon 4$ heterozygote $(\varepsilon 2 / \varepsilon 4, \varepsilon 3 / \varepsilon 4)$ and $\varepsilon 4$ homozygosis $(\varepsilon 4 / \varepsilon 4)$ exhibited a significant correlation with an increased risk of IS. Notably, OR in $\varepsilon 4$ homozygosis ( $\varepsilon 4 / \varepsilon 4$ vs. $\varepsilon 3 / 3: 1.833$ (95\% CI $1.542-2.179)$ ) was higher than those in $\varepsilon 4$ heterozygotes $(\varepsilon 2 / \varepsilon 4$ vs. $\varepsilon 3 / 3$ : 1.233 (95\% CI $1.056-1.440)$ and $\varepsilon 3 / \varepsilon 4$ vs. $\varepsilon 3 / 3: 1.340$ (95\% CI 1.165-1.542)), which implied that the $\varepsilon 4$ allele might possess a cumulative effect. Then, we performed comparisons between $\varepsilon 4 / \varepsilon 4$ and $\varepsilon 2 / \varepsilon 4$ or $\varepsilon 3 / \varepsilon 4$; there existed significant differences between $\varepsilon 4$ homozygosis and $\varepsilon 4$ heterozygote. The OR between $\varepsilon 4 / \varepsilon 4$ and $\varepsilon 2 / \varepsilon 4$ was 1.625 (95\% CI 1.281-2.060, $P<0.0001)$; the OR between $\varepsilon 4 / \varepsilon 4$ and $\varepsilon 3 / \varepsilon 4$ was 1.301 (95\% CI 1.077-1.571, $P=0.01$ ), giving a hint that $\varepsilon 4$ homozygosis might bring a higher risk of IS than $\varepsilon 4$ heterozygotes.

There are tremendous researches and discussions focusing on the pathogenicity of $\varepsilon 4$. An Indian research reported that VLDL and triglycerides levels were found to be significantly associated with $\varepsilon 2 / \varepsilon 4$ and $\varepsilon 3 / \varepsilon 4$ genotypes; the $\varepsilon 4$ allele exerted a higher influence than the $\varepsilon 3$ allele in plasma cholesterol levels [22]. As a lipid transport protein, APOE3 and APOE2 preferentially bind to the smaller, more phospholipid-enriched high-density lipoproteins (HDL), while APOE4 preferentially binds to the larger, triglyceride-rich very low-density lipoproteins (VLDL). Miyata and Smith demonstrated an antioxidant activity in the order APOE2 $>\mathrm{E} 3>\mathrm{E} 4$, and other researchers also reported similar results that APOE4 was associated with increased oxidative stress $[25,80]$, which might play a role in atherosclerosis and lead to increased risk of ischemic vascular diseases. Besides the above reasons, APOE4 was proved to be neurotoxic by assuming an abnormal conformation (the unique domain interaction between Arg-61 and Glu-255) which was highly susceptible to neuron specific proteolysis and generating neurotoxic fragments that escaped the secretory pathway and entered the cytosol [81]. Totally, from pathophysiological mechanisms to clinical research results, it seems that APOE4 is indeed related to a higher risk of IS, compared with other isoforms, both in $\varepsilon 4$ heterozygote and homozygous. $\varepsilon 2$ allele appears to be unclear and controversial in stroke [13]. In a meta-analysis of Martínez-González et al., compared with $\varepsilon 3 / \varepsilon 3$, APOE $\varepsilon 2$ was associated with intracerebral hemorrhage
$(\mathrm{OR}=1.32 ; 95 \% \mathrm{CI}, 1.01-1.74)$; meanwhile, APOE $\varepsilon 2$ was more related to lobar hemorrhage than deep hemorrhage [82]. As to the association of IS with APOE based on previous investigation, it is uncertain. Our estimates showed that both $\varepsilon 2 / \varepsilon 2$ and $\varepsilon 2 / \varepsilon 3$ genotypes exhibited no significant effects on IS risk, compared with $\varepsilon 3 / \varepsilon 3$. Also, no differences were found in comparisons of $\varepsilon 2$ allele vs. $\varepsilon 3$ allele and $\varepsilon 2$ vs. non- $\varepsilon 2$ carriers. This result remained consistent with another meta-analysis in 2013 [14]. Interestingly, in subtype analysis, $\varepsilon 2 / \varepsilon 2$ displayed significances in the $\mathrm{CE}$ group $(\mathrm{OR}=4.290 ; 95 \% \mathrm{CI}, 1.917-9.600 ; P<0.0001)$ and SAD group (OR $=1.803 ; 95 \% \mathrm{CI}, 1.037-3.134 ; P=0.04)$. The largest meta-analysis of the APOE genotype with IS showed a positive linear association of increasing risk when ordered from $\varepsilon 2 / \varepsilon 2, \varepsilon 2 / \varepsilon 3, \varepsilon 2 / \varepsilon 4, \varepsilon 3 / \varepsilon 3, \varepsilon 3 / \varepsilon 4$, and $\varepsilon 4 / \varepsilon 4$ in European ancestry population [83]. The conclusion might explain why APOE4 brings a higher risk of IS but could not clarify that the CE and SAD subgroups in comparison of $\varepsilon 2 /$ $\varepsilon 2$ with $\varepsilon 3 / \varepsilon 3$ show significances. It is well known that all patients with type III hyperlipidemia (dysbetalipoproteinemia) were APOE $\varepsilon 2$ homozygous, whereas most $\varepsilon 2 /$ $\varepsilon 2$ subjects ( $>90 \%)$ were normolipidemic or even hypolipidemic, owing to reductions in LDL or HDL or both. Therefore, the APOE $\varepsilon 2$ allele has both increased and decreased risks for atherosclerosis, which induced a comprehensive and undetermined result [84].

As to our subtype analyses, all LAA groups showed no significant difference among comparisons, which raised a question why isoforms of APOE, a lipid transport protein, seemed not to be related with IS caused by large artery atherosclerosis. Besides lipid metabolism and atherosclerosis, there might exist some other pathways underlying the relationships between APOE and risk of IS. Our estimates displayed that APOE isoforms were associated to risk of IS especially in the SAD subgroup. Hypertension was known to be an independent risk factor of SAD. Atherosclerosis, dyslipidemia, and hypertension have a complex interaction, and the causations with APOE need further investigation.

Our meta-analysis has several limitations. First, just as the abovementioned, heterogeneity between studies remains undeterminable. Second, results of our meta-analysis based on case control studies cannot provide a causal relationship, but only an association. Third, age variable and ethnicity can influence APOE frequencies in a population; we cannot obtain sufficient related information to perform further subdivided subgroup analyses. Fourth, other pathogenic factors about IS, a multifactorial disease, such as plasma lipid levels, hypertension, life-style, BMI, and gene-environment interactions, were unachievable. Fifth, the controls in accessible studies were not strictly defined; some were selected from healthy populations and others were from nonstroke people. The expected genotype distribution in controls was not in accordance with HWE in seven studies. Population selection in control groups failed to avoid certain diseases which might have a relation with the APOE gene, such as dyslipidemia, hypertension, other vascular diseases, and diabetes. Sixth, the case groups were not selected by a prospective process and the design of case control studies often caused abnormal gene frequency. 


\section{Conclusions}

In conclusion, our meta-analysis provides rational evidence that APOE $\varepsilon 4$ mutation is a genetic risk factor for IS. Prospective studies of a large sample size, which concerns gene-gene and gene-environment interactions, should be carried out in the future to reach a more comprehensive outcome about the association of APOE gene polymorphisms and IS. What is more, future researches should be designed to elucidate the mechanism by which APOE $\varepsilon 4$ mutation adds the risk of IS.

\section{Data Availability}

Data presented within the paper and the supplementary materials contributed to the findings in our study. They are all are available from our corresponding author for reasonable request.

\section{Conflicts of Interest}

The authors declare no conflict of interest.

\section{Authors' Contributions}

The conceptualization was done by S.-Y. Q., K. S., Y.-H.C., and X.C.; the methodology was done by D.-S. T., D.-J. P., and C. Q.; K. S., Y-H.C., H.-H. Y, and X.C. took care of the software; meta-analysis was done by D.-S. T., D.-J. P., C. Q., S.-Y. Q., K. S., and X.C.; writing-original draft preparation-was done by S.-Y. Q., K. S., Y.-H.C., and H.-H. Y.; writing-review and editing-was done by D.-S. T., D.J. P., and C. Q. All authors have read and agreed to the published version of the manuscript. Su-Ya Qiao and Ke Shang contributed equally to this work.

\section{Acknowledgments}

We are deeply grateful for all our colleagues of the Department of Neurology in Tongji Hospital.

\section{Supplementary Materials}

Supplementary material Table 1: fifteen of the included studies provide data about different subtypes of IS: LAA, SAD, and CE. Supplementary material Table 2: Newcastle-Ottawa Scale (NOS) score of included studies. Supplementary material Table 3: PRISMA list of our meta-analysis. Supplementary material Table 4: sensitivity analysis of the association between ApoE gene polymorphisms and IS. Supplementary material Table 5: publication bias and heterogeneity of our meta-analysis. Supplementary material Figure 1: funnel plots for studies included in Figures 2A-G. Supplementary material Figure 2: funnel plots for studies included in Figures 3A-G. Supplementary material Figure 3: results of meta-regression. (Supplementary Materials)

\section{References}

[1] R. Bonita, "Epidemiology of stroke," Lancet (London, England), vol. 339, no. 8789, pp. 342-344, 1992.
[2] A. Munshi and S. Kaul, "Genetic basis of stroke: an overview," Neurology India, vol. 58, no. 2, pp. 185-190, 2010.

[3] X. Wang, S. Cheng, V. H. Brophy et al., "A meta-analysis of candidate gene polymorphisms and ischemic stroke in 6 study populations: association of lymphotoxin-alpha in nonhypertensive patients," Stroke, vol. 40, no. 3, pp. 683-695, 2009.

[4] A. D. Marais, "Apolipoprotein E in lipoprotein metabolism, health and cardiovascular disease," Pathology, vol. 51, no. 2, pp. 165-176, 2019.

[5] R. W. Mahley, "Apolipoprotein E: cholesterol transport protein with expanding role in cell biology," Science (New York, N.Y.), vol. 240, no. 4852, pp. 622-630, 1988.

[6] M. Jo, T. Silke, N. Katherine et al., "Low-density lipoprotein receptor deficiency attenuates neuroinflammation through the induction of apolipoprotein E," Frontiers in Immunology, vol. 8, 2017.

[7] L. Li, R. Li, A. Zacharek et al., “ABCA1/ApoE/HDL signaling pathway facilitates myelination and oligodendrogenesis after stroke," International Journal of Molecular Sciences, vol. 21, no. 12, p. 4369, 2020.

[8] A. Montagne, D. A. Nation, A. P. Sagare et al., “_APOE4_ leads to blood -brain barrier dysfunction predicting cognitive decline," Nature, vol. 581, no. 7806, pp. 71-76, 2020.

[9] M. Granér, J. Kahri, M. Varpula et al., “Apolipoprotein E polymorphism is associated with both carotid and coronary atherosclerosis in patients with coronary artery disease," Nutrition, Metabolism, and Cardiovascular Diseases: NMCD, vol. 18, no. 4, pp. 271-277, 2008.

[10] Y. Ruixing, W. Jinzhen, L. Weixiong, C. Yuming, Y. Dezhai, and P. Shangling, "The environmental and genetic evidence for the association of hyperlipidemia and hypertension," Journal of Hypertension, vol. 27, no. 2, pp. 251-258, 2009.

[11] A. L. Lumsden, A. Mulugeta, A. Zhou, and E. Hypponen, "Apolipoprotein E (APOE) genotype-associated disease risks: a phenome-wide, registry-based, case-control study utilising the UK Biobank," eBioMedicine, vol. 59, p. 102954, 2020.

[12] L. Cattin, M. Fisicaro, M. Tonizzo et al., "Polymorphism of the apolipoprotein E gene and early carotid atherosclerosis defined by ultrasonography in asymptomatic adults," Arteriosclerosis, Thrombosis, and Vascular Biology, vol. 17, no. 1, pp. 91-94, 1997.

[13] M. O. McCarron, D. Delong, and M. J. Alberts, “APOE genotype as a risk factor for ischemic cerebrovascular disease: a meta-analysis," Neurology, vol. 53, no. 6, pp. 1308-1311, 1999.

[14] Q. Y. Wang, W. J. Wang, L. Wu, L. Liu, and L. Z. Han, "Metaanalysis of APOE $\varepsilon 2 / \varepsilon 3 / \varepsilon 4$ polymorphism and cerebral infarction," Journal of Neural Transmission (Vienna), vol. 120, no. 10, pp. 1479-1489, 2013.

[15] C. Chen and Z. Hu, "ApoE polymorphisms and the risk of different subtypes of stroke in the Chinese population: a comprehensive meta-analysis," Cerebrovascular Diseases, vol. 41, no. 3-4, pp. 119-138, 2016.

[16] D. Moher, A. Liberati, J. Tetzlaff, and D. G. Altman, "Preferred reporting items for systematic reviews and meta-analyses: the PRISMA statement," Journal of Clinical Epidemiology, vol. 62, no. 10, pp. 1006-1012, 2009.

[17] J. P. Higgins, S. G. Thompson, J. J. Deeks, and D. G. Altman, "Measuring inconsistency in meta-analyses," BMJ (Clinical Research Ed.), vol. 327, no. 7414, pp. 557-560, 2003.

[18] J. Meng, S. Wang, M. Zhang, S. Fan, L. Zhang, and C. Liang, "TP73 G4C14-A4T14polymorphism and cancer susceptibility: 
evidence from 36 case-control studies," Bioscience Reports, vol. 38 , no. 6,2018 .

[19] H. Wu, Q. Huang, Z. Yu, H. Wu, and Z. Zhong, "The SNPs rs429358 and rs7412 of APOE gene are association with cerebral infarction but not SNPs rs2306283 and rs4149056 of SLCO1B1 gene in southern Chinese Hakka population," Lipids in Health and Disease, vol. 19, no. 1, p. 202, 2020.

[20] L. L. Zhao, G. Su, L. X. Chen et al., “Apolipoprotein E polymorphisms are associated with ischemic stroke susceptibility in a Northwest China Han population," Bioscience Reports, vol. 37, no. 6, 2017.

[21] D. Coen Herak, J. Lenicek Krleza, M. Radic Antolic et al., "Association of polymorphisms in coagulation factor genes and enzymes of homocysteine metabolism with arterial ischemic stroke in children," Clinical and Applied Thrombosis/ Hemostasis, vol. 23, no. 8, pp. 1042-1051, 2017.

[22] S. Das, S. Kaul, A. Jyothy, and A. Munshi, "Association of APOE (E2, E3 and E4) gene variants and lipid levels in ischemic stroke, its subtypes and hemorrhagic stroke in a south Indian population," Neuroscience Letters, vol. 628, pp. 136141, 2016.

[23] C. Koopal, M. I. Geerlings, M. Muller et al., "The relation between apolipoprotein E (_APOE_) genotype and peripheral artery disease in patients at high risk for cardiovascular disease," Atherosclerosis, vol. 246, pp. 187-192, 2016.

[24] M. Luo, J. Li, X. Sun et al., "Interactions among candidate genes selected by meta-analyses resulting in higher risk of ischemic stroke in a Chinese population," PLoS One, vol. 10, no. 12, article e0145399, 2015.

[25] L. K. Wei, A. Au, S. Menon, S. H. Gan, and L. R. Griffiths, "Clinical relevance of MTHFR, eNOS, ACE, and ApoE gene polymorphisms and serum vitamin profile among Malay patients with ischemic stroke," Journal of Stroke and Cerebrovascular Diseases, vol. 24, no. 9, pp. 2017-2025, 2015.

[26] H. Q. Yan, Y. Yuan, P. Zhang, Z. Huang, L. Chang, and Y. K. Gui, "Association of the ApoE gene polymorphism and dietary factors with cerebral infarction and circulating lipid concentrations," Genetics and Molecular Research, vol. 14, no. 1, pp. 665-670, 2015.

[27] D. Chatzistefanidis, S. Giannopoulos, K. Spengos et al., "Apolipoprotein E polymorphisms and ischaemic stroke: a twocenter Greek study," European Journal of Neurology, vol. 21, no. 8, pp. 1083-1088, 2014.

[28] M. Atadzhanov, M. H. Mwaba, P. N. Mukomena et al., "Association of the APOE, MTHFR and ACE genes polymorphisms and stroke in Zambian patients," Neurology International, vol. 5, no. 4, article e20, 2013.

[29] A. A. Gelfand, L. A. Croen, A. R. Torres, and Y. W. Wu, "Genetic risk factors for perinatal arterial ischemic stroke," Pediatric Neurology, vol. 48, no. 1, pp. 36-41, 2013.

[30] A. Balcerzyk, I. Zak, P. Niemiec et al., “_APOE_ Gene $\varepsilon$ Polymorphism Does Not Determine Predisposition to Ischemic Stroke in Children," Pediatric Neurology, vol. 43, no. 1, pp. 25-28, 2010.

[31] Y. Tamam, N. Tasdemir, R. Toprak, B. Tamam, and K. Iltumur, "Apolipoprotein E genotype in patients with cerebrovascular diseases and its effect on the disease outcome," The International Journal of Neuroscience, vol. 119, no. 7, pp. 919-935, 2009.

[32] N. Tascilar, A. Dursun, H. Ankarali et al., "Relationship of apoE polymorphism with lipoprotein (a), apoA, apoB and lipid levels in atherosclerotic infarct," Journal of the Neurological Sciences, vol. 277, no. 1-2, pp. 17-21, 2009.

[33] B. Wang, H. Zhao, L. Zhou et al., "Association of genetic variation in apolipoprotein $\mathrm{E}$ and low density lipoprotein receptor with ischemic stroke in northern Han Chinese," Journal of the Neurological Sciences, vol. 276, no. 1-2, pp. 118-122, 2009.

[34] C. L. Lai, C. K. Liu, R. T. Lin, and C. T. Tai, "Association of apolipoprotein E polymorphism with ischemic stroke subtypes in Taiwan," The Kaohsiung Journal of Medical Sciences, vol. 23, no. 10, pp. 491-497, 2007.

[35] M. G. Parfenov, T. Y. Nikolaeva, M. A. Sudomoina et al., "Polymorphism of apolipoprotein E (APOE) and lipoprotein lipase (LPL) genes and ischaemic stroke in individuals of Yakut ethnicity," Journal of the Neurological Sciences, vol. 255, no. 1-2, pp. 42-49, 2007.

[36] S. Y. Kang and W. I. Lee, "Apolipoprotein e polymorphism in ischemic stroke patients with different pathogenetic origins," The Korean Journal of Laboratory Medicine, vol. 26, no. 3, pp. 210-216, 2006.

[37] X. Gao, H. Yang, and T. ZhiPing, "Association studies of genetic polymorphism, environmental factors and their interaction in ischemic stroke," Neuroscience Letters, vol. 398, no. 3, pp. 172-177, 2006.

[38] L. Baum, H. K. Ng, K. S. Wong et al., “Associations of apolipoprotein E exon 4 and lipoprotein lipase S447X polymorphisms with acute ischemic stroke and myocardial infarction," Clinical Chemistry and Laboratory Medicine, vol. 44, no. 3, pp. 274281, 2006.

[39] A. Pezzini, M. Grassi, E. Del Zotto et al., "Cumulative effect of predisposing genotypes and their interaction with modifiable factors on the risk of ischemic stroke in young adults," Stroke, vol. 36, no. 3, pp. 533-539, 2005.

[40] P. Cerrato, C. Baima, M. Grasso et al., "Apolipoprotein E polymorphism and stroke subtypes in an Italian cohort," Cerebrovascular Diseases, vol. 20, no. 4, pp. 264-269, 2005.

[41] Z. Q. Jin, Y. S. Fan, J. Ding et al., "Association of apolipoprotein E 4 polymorphism with cerebral infarction in Chinese Han population," Acta Pharmacologica Sinica, vol. 25, no. 3, pp. 352-356, 2004.

[42] S. Duzenli, I. Pirim, A. Gepdiremen, and O. Deniz, "Apolipoprotein E polymorphism and stroke in a population from eastern Turkey," Journal of Neurogenetics, vol. 18, no. 1, pp. 365-375, 2004.

[43] A. Slowik, T. Iskra, W. Turaj, J. Hartwich, A. Dembinska-Kiec, and A. Szczudlik, "LDL phenotype B and other lipid abnormalities in patients with large vessel disease and small vessel disease," Journal of the Neurological Sciences, vol. 214, no. 12, pp. 11-16, 2003.

[44] D. R. Souza, B. F. Campos, E. F. Arruda, L. J. Yamamoto, D. M. Trindade, and W. A. Tognola, "Influence of the polymorphism of apolipoprotein E in cerebral vascular disease," Arquivos de Neuro-Psiquiatria, vol. 61, no. 1, pp. 7-13, 2003.

[45] V. Karttunen, G. Alfthan, L. Hiltunen et al., "Risk factors for cryptogenic ischaemic stroke," European Journal of Neurology, vol. 9, no. 6, pp. 625-632, 2002.

[46] A. C. Morrison, C. M. Ballantyne, M. Bray, L. E. Chambless, A. R. Sharrett, and E. Boerwinkle, "LPL polymorphism predicts stroke risk in men," Genetic Epidemiology, vol. 22, no. 3, pp. 233-242, 2002.

[47] M. J. MacLeod, R. P. De Lange, G. Breen, D. Meiklejohn, H. Lemmon, and D. S. Clair, "Lack of association between 
apolipoprotein E genoype and ischaemic stroke in a Scottish population," European Journal of Clinical Investigation, vol. 31, no. 7, pp. 570-573, 2001.

[48] A. H. Chowdhury, T. Yokoyama, Y. Kokubo, M. M. Zaman, A. Haque, and $\mathrm{H}$. Tanaka, "Apolipoprotein E genetic polymorphism and stroke subtypes in a Bangladeshi hospital-based study," Journal of Epidemiology, vol. 11, no. 3, pp. 131-138, 2001.

[49] R. Frikke-Schmidt, B. G. Nordestgaard, D. Thudium, M. L. Moes Grønholdt, and A. Tybjaerg-Hansen, "APOE genotype predicts $\mathrm{AD}$ and other dementia but not ischemic cerebrovascular disease," Neurology, vol. 56, no. 2, pp. 194-200, 2001.

[50] A. J. Catto, L. J. McCormack, M. W. Mansfield et al., "Apolipoprotein E polymorphism in cerebrovascular disease," Acta Neurologica Scandinavica, vol. 101, no. 6, pp. 399-404, 2000.

[51] Y. Kokubo, A. H. Chowdhury, C. Date, T. Yokoyama, H. Sobue, and H. Tanaka, "Age-dependent association of apolipoprotein E genotypes with stroke subtypes in a Japanese rural population," Stroke, vol. 31, no. 6, pp. 12991306, 2000.

[52] D. Q. Peng, S. P. Zhao, and J. L. Wang, "Lipoprotein (a) and apolipoprotein E $\varepsilon 4$ as independent risk factors for ischemic stroke," Journal of Cardiovascular Risk, vol. 6, no. 1, pp. 1-6, 1999.

[53] Y. Ji, K. Urakami, Y. Adachi, M. Maeda, K. Isoe, and K. Nakashima, "Apolipoprotein E polymorphism in patients with Alzheimer's disease, vascular dementia and ischemic cerebrovascular disease," Dementia and Geriatric Cognitive Disorders, vol. 9, no. 5, pp. 243-245, 1998.

[54] M. Margaglione, D. Seripa, C. Gravina et al., "Prevalence of apolipoprotein E alleles in healthy subjects and survivors of ischemic stroke: an Italian case-control study," Stroke, vol. 29, no. 2, pp. 399-403, 1998.

[55] C. Kessler, C. Spitzer, D. Stauske et al., "The apolipoprotein E and beta-fibrinogen G/A-455 gene polymorphisms are associated with ischemic stroke involving large-vessel disease," Arteriosclerosis, Thrombosis, and Vascular Biology, vol. 17, no. 11, pp. 2880-2884, 1997.

[56] V. Hachinski, C. Graffagnino, M. Beaudry et al., "Lipids and Stroke," Archives of Neurology, vol. 53, no. 4, pp. 303-308, 1996.

[57] R. Couderc, F. Mahieux, S. Bailleul, G. Fenelon, R. Mary, and J. Fermanian, "Prevalence of apolipoprotein E phenotypes in ischemic cerebrovascular disease. A case-control study," Stroke, vol. 24, no. 5, pp. 661-664, 1993.

[58] L. Qian, L. Ding, L. Cheng et al., "Early biomarkers for poststroke cognitive impairment," Journal of Neurology, vol. 259, no. 10, pp. 2111-2118, 2012.

[59] C. Konialis, K. Spengos, P. Iliopoulos et al., "The APOE E4 allele confers increased risk of ischemic stroke among Greek carriers," Advances in Clinical and Experimental Medicine, vol. 25, no. 3, pp. 471-478, 2016.

[60] H. A. Fayed, E. A. Seidy, E. Shawki, H. A. Serogy, and M. A. J. E. Attia, "Neurosurgery, The risk and prognostic value of apolipoprotein E gene polymorphism in stroke," The Egyptian Journal of Neurology, Psychiatry and Neurosurgery, vol. 46, no. 2, pp. 541-550, 2009.

[61] S. Stankovic, Z. Jovanovic-Markovic, N. Majkic-Singh et al., "Apolipoprotein e gene polymorphism as a risk factor for ischemic cerebrovascular disease," Jugoslovenska medicinska biohemija, vol. 23, no. 3, pp. 255-264, 2004.
[62] J. Pedro-Botet, M. Sentí, X. Nogués et al., "Lipoprotein and apolipoprotein profile in men with ischemic stroke. Role of lipoprotein (a), triglyceride-rich lipoproteins, and apolipoprotein E polymorphism," Stroke, vol. 23, no. 11, pp. 1556-1562, 1992.

[63] N. Fekih-Mrissa, S. Klai, M. Mrad et al., "Apolipoprotein E genotypes associated with Alzheimer disease and concomitant stroke," Journal of Stroke and Cerebrovascular Diseases, vol. 23, no. 4, pp. 681-685, 2014.

[64] J. N. Brewin, A. Smith, R. Cook et al., "Genetic Analysis of Patients with Sickle Cell Anemia and Stroke before 4 Years of Age Suggest an Important Role for Apoliprotein E," Circulation. Genomic and precision medicine, vol. 13, no. 5, pp. 531-540, 2020.

[65] S. Saidi, W. Zammiti, L. B. Slamia, S. B. Ammou, W. Y. Almawi, and T. Mahjoub, "Interaction of angiotensinconverting enzyme and apolipoprotein E gene polymorphisms in ischemic stroke involving large-vessel disease," Journal of Thrombosis and Thrombolysis, vol. 27, no. 1, pp. 68-74, 2009.

[66] H. M. Wen, L. Baum, W. S. Cheung et al., “Apolipoprotein E epsilon4 allele is associated with the volume of white matter changes in patients with lacunar infarcts," European Journal of Neurology, vol. 13, no. 11, pp. 1216-1220, 2006.

[67] G. Giassakis, S. Veletza, N. Papanas, I. Heliopoulos, and H. Piperidou, "Apolipoprotein E and first-ever ischaemic stroke in Greek hospitalized patients," The Journal of International Medical Research, vol. 35, no. 1, pp. 127-133, 2007.

[68] Y. Nakata, T. Katsuya, H. Rakugi et al., "Polymorphism of angiotensin converting enzyme, angiotensinogen, and apolipoprotein E genes in a Japanese population with cerebrovascular disease," American Journal of Hypertension, vol. 10, no. 12, pp. 1391-1395, 1997.

[69] Z. Szolnoki, F. Somogyvari, A. Kondacs, M. Szabo, and L. Fodor, "Evaluation of the interactions of common genetic mutations in stroke subtypes," Journal of Neurology, vol. 249, no. 10, pp. 1391-1397, 2002.

[70] K. Aalto-Setala, H. Palomaki, H. Miettinen et al., "Genetic risk factors and ischaemic cerebrovascular disease: role of common variation of the genes encoding apolipoproteins and angiotensin-converting enzyme," Annals of Medicine, vol. 30, no. 2, pp. 224-233, 1998.

[71] M. Artieda, A. Gañán, A. Cenarro et al., "Association and linkage disequilibrium analyses of APOE polymorphisms in atherosclerosis," Disease Markers, vol. 24, no. 2, 2008.

[72] J. A. Schneider, J. L. Bienias, R. S. Wilson, E. Berry-Kravis, D. A. Evans, and D. A. Bennett, "The apolipoprotein E epsilon4 allele increases the odds of chronic cerebral infarction [corrected] detected at autopsy in older persons," Stroke, vol. 36, no. 5, pp. 954-959, 2005.

[73] X. B. Li, J. Wang, A. D. Xu et al., “Apolipoprotein E polymorphisms increase the risk of post-stroke depression," Neural Regeneration Research, vol. 11, no. 11, pp. 1790-1796, 2016.

[74] S. Fan, J. Meng, L. Zhang, X. Zhang, and C. Liang, "CAV1 polymorphisms rs1049334, rs1049337, rs7804372 might be the potential risk in tumorigenicity of urinary cancer: a systematic review and meta-analysis," Pathology, Research and Practice, vol. 215, no. 1, pp. 151-158, 2019.

[75] S. L. Gao, Y. D. Chen, C. Yue et al., “-196 to $-174 \mathrm{del}$, rs4696480, rs3804099 polymorphisms of Toll-like receptor 2 gene impact the susceptibility of cancers: evidence from 37053 subjects," Bioscience Reports, vol. 39, no. 12, 2019. 
[76] P. B. Verghese, J. M. Castellano, and D. M. Holtzman, “Apolipoprotein E in Alzheimer's disease and other neurological disorders," The Lancet. Neurology, vol. 10, no. 3, pp. 241-252, 2011.

[77] A. Serrano-Pozo, S. Das, and B. T. Hyman, "APOE and Alzheimer's disease: advances in genetics, pathophysiology, and therapeutic approaches," The Lancet. Neurology, vol. 20, no. 1, pp. 68-80, 2021.

[78] S. S. Mirza, U. Saeed, J. Knight et al., “APOE\&4, white matter hyperintensities, and cognition in Alzheimer and Lewy body dementia," Neurology, vol. 93, no. 19, pp. e1807-e1819, 2019.

[79] A. Charidimou, H. I. Zonneveld, S. Shams et al., "APOEand cortical superficial siderosis in CAA," Neurology, vol. 93, no. 4, pp. e358-e371, 2019.

[80] M. Miyata and J. D. Smith, “Apolipoprotein E allele-specific antioxidant activity and effects on cytotoxicity by oxidative insults and beta-amyloid peptides," Nature Genetics, vol. 14, no. 1, pp. 55-61, 1996.

[81] R. W. Mahley and Y. Huang, "Small-molecule structure correctors target abnormal protein structure and function: structure corrector rescue of apolipoprotein E4-associated neuropathology," Journal of Medicinal Chemistry, vol. 55, no. 21, pp. 8997-9008, 2012.

[82] N. A. Martínez-González and C. L. Sudlow, "Effects of apolipoprotein E genotype on outcome after ischaemic stroke, intracerebral haemorrhage and subarachnoid haemorrhage," Journal of Neurology, Neurosurgery, and Psychiatry, vol. 77, no. 12, pp. 1329-1335, 2006.

[83] T. A. Khan, T. Shah, D. Prieto et al., “Apolipoprotein E genotype, cardiovascular biomarkers and risk of stroke: systematic review and meta-analysis of 14,015 stroke cases and pooled analysis of primary biomarker data from up to 60,883 individuals," International journal of epidemiology, vol. 42, no. 2 , pp. 475-492, 2013.

[84] R. W. Mahley and S. C. Rall Jr., “Apolipoprotein E: far more than a lipid transport protein," Annual Review of Genomics and Human Genetics, vol. 1, pp. 507-537, 2000. 